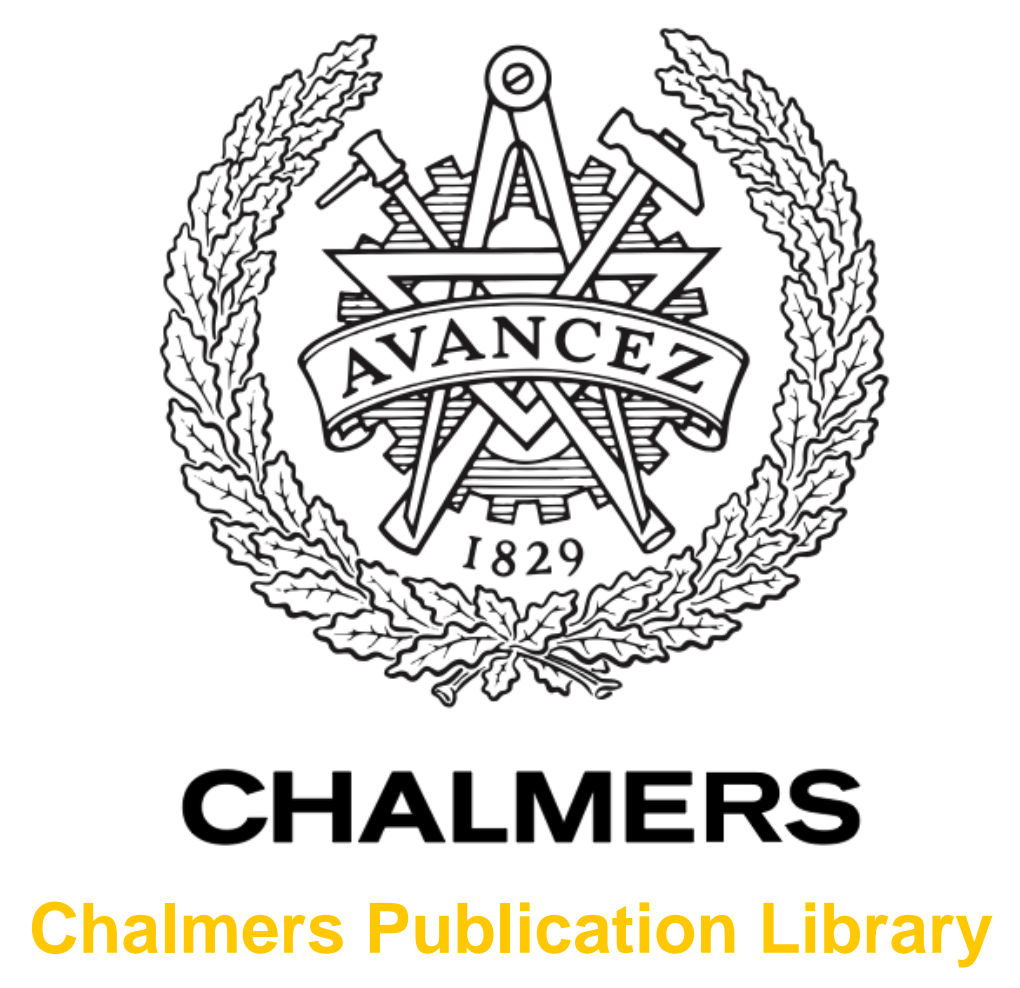

\title{
Embedded Large-Eddy Simulation Using the Partially Averaged Navier-Stokes Model
}

This document has been downloaded from Chalmers Publication Library (CPL). It is the author's version of a work that was accepted for publication in:

AIAA Journal (ISSN: 0001-1452)

Citation for the published paper:

Davidson, L. ; Peng, S. (2013) "Embedded Large-Eddy Simulation Using the Partially

Averaged Navier-Stokes Model". AIAA Journal, vol. 51(5), pp. 1066-1079.

http://dx.doi.org/10.2514/1.J051864

Downloaded from: http://publications.lib.chalmers.se/publication/174847

Notice: Changes introduced as a result of publishing processes such as copy-editing and formatting may not be reflected in this document. For a definitive version of this work, please refer to the published source. Please note that access to the published version might require a subscription.

Chalmers Publication Library (CPL) offers the possibility of retrieving research publications produced at Chalmers University of Technology. It covers all types of publications: articles, dissertations, licentiate theses, masters theses, conference papers, reports etc. Since 2006 it is the official tool for Chalmers official publication statistics. To ensure that Chalmers research results are disseminated as widely as possible, an Open Access Policy has been adopted.

The CPL service is administrated and maintained by Chalmers Library. 


\title{
Embedded Large-Eddy Simulation Using the Partially
}

\section{Averaged Navier-Stokes Model}

\author{
Lars Davidson ${ }^{1 *}$ \\ ${ }^{1}$ Department of Applied Mechanics, Chalmers University of Technology. \\ SE-412 96 Gothenburg, SWEDEN \\ Shia-Hui Peng ${ }^{1,2 *}$ \\ ${ }^{2}$ FOI, Swedish Defence Research Agency, SE-164 90, Stockholm, SWEDEN
}

\begin{abstract}
An embedded LES modelling approach is explored and verified using the PANS (Partially Averaged Navier-Stokes) model as a platform. With the same base model, the turbulence-resolving LES region is embedded by setting the PANS model coefficient to $f_{k}<1$ as distinguished from its neighboring RANS region, where $f_{k}=1$ is specified. The embedded LES approach is verified in computations of a turbulent channel flow and a turbulent flow over a hump. Emphasis is placed on the impact of turbulent conditions at the RANS-LES interface using anisotropic velocity fluctuations generated from synthetic turbulence. The effect of the spanwise size of the computational domain is investigated. It is shown that the embedded LES method based on the PANS modelling approach is computationally feasible and able to provide reasonable turbulence-resolving predictions in the embedded LES region. The WALE (Wall-adapting local eddy-viscosity) model is also evaluated for the hump flow and it is found that its performance is worse than that of the the LRN (Low-Reynolds Number) PANS model when the results are compared with experiments.
\end{abstract}

*Professor, Department of Applied Mechanics, Chalmers University of Technology 


\section{Nomenclature}

\section{Roman Symbols}

$B, D, y_{b l}$ constants in Eq. 16

c hump length, see Fig. 2

$C_{\mu}, C_{\varepsilon 1}, C_{\varepsilon 2}, C_{\varepsilon 2}^{*}$ constants in the turbulence model

$C_{f} \quad$ skin friction $\left(=\tau_{w} /\left(0.5 \rho U_{i n}^{2}\right)\right)$

$C_{p} \quad$ pressure coefficient $\left(=\left(p_{\text {outlet }}-p\right) /\left(0.5 \rho U_{\text {in }}^{2}\right)\right)$

$C_{S} \quad$ Smagorinsky constant

$f_{\mu}, f_{2}$ damping functions in the turbulence model

$f_{k}, f_{\varepsilon}$ ratio of resolved to total of, respectively, $k$ and $\varepsilon$

$f_{b l} \quad$ blending function, see Eq. 16

$H \quad$ channel height, see Fig. 2

$h \quad$ hump height, see Fig. 2

$k \quad$ turbulent kinetic energy

$\ell \quad$ turbulent length scale

$P \quad$ production

$p \quad$ pressure

Re Reynolds number

$t \quad$ time

$U, V, W$ time-averaged velocity in $x, y$ and $z$ direction, respectively

$u, v, w$ velocity in $x, y$ and $z$ direction, respectively

$u_{\tau} \quad$ wall-friction velocity $\left(=\sqrt{\tau_{w} / \rho}\right)$

$u_{i} \quad$ velocity in $x_{i}$ direction

V volume

$x, y, z$ Cartesian coordinate directions, see Figs. 1 and 2

$x_{i} \quad$ Cartesian coordinate vector

y wall-normal coordinate direction or distance

$y^{*} \quad$ non-dimensional wall distance 


$$
\begin{array}{ll}
Z_{\max } & \text { spanwise extent } \\
\mathcal{L} & \text { integral length scale } \\
\mathcal{T} & \text { integral time scale } \\
\mathcal{U}^{\prime} & \text { synthetic inlet fluctuation }
\end{array}
$$

\section{Subscripts}

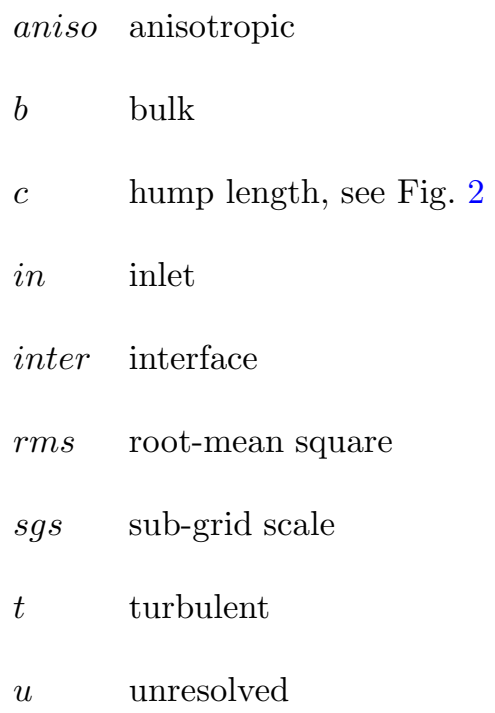




\section{Introduction}

It is well known that LES (Large Eddy Simulation) may become prohibitively costly when applied to wallbounded turbulent flows at high Reynolds numbers because of the requirement of near-wall grid resolution. To circumvent this requirement, much research over the past 15 years has been dedicated to the development of DES (Detached Eddy Simulation) and other similar hybrid LES-RANS methods in which the near-wall region is treated with RANS (Reynolds-Averaged Navier-Stokes) and the remaining region is covered with LES. In these methods, the RANS-LES coupling usually occurs over a surface parallel to the wall.

Another modelling approach that combines LES and RANS is embedded LES, in which an LES region is embedded in any desired region, coupled with surrounding RANS simulations, and the location of the RANS-LES interaction is specified, for example, over a surface normal to the streamwise direction, as shown in Figs. 1 and 2(a). Regions with flow separation and vortex motions are typically treated using embedded LES, while the rest of the computational domain is accommodated by RANS.

The work of Quéméré and Sagaut ${ }^{1}$ is one of the earliest on embedded LES. They computed the flow over a blunt trailing edge where LES was used in the wake region. The turbulent fluctuations at the RANS-LES interface were taken from a precursor LES simulation of channel flow. Batten et al. ${ }^{2}$ used a LNS (Limited-Numerical-Scale) method which can operate both in RANS and LES mode. At the RANSLES interface synthetic fluctuations were added to stimulate the energy transfer from modelled to resolved turbulence. They validated their method in channel flow. Terracol ${ }^{3}$ used zonal RANS-LES modelling to predict the flow around an airfoil, with the intention of developing a method for predicting trailing edge noise. 2D RANS was used in the entire domain, and an LES region with a small spanwise extent was used in the wake region. Two methods for generating turbulent fluctuations at the RANS-LES interface were evaluated, namely, the recycling method and synthetic fluctuations. It was concluded that synthetic fluctuations were preferable since the recycling method introduced artificial streamwise periodic fluctuations. Mathey and Cokljat ${ }^{4}$ studied the flow around the Ahmed body using embedded LES. The flow around the 
entire body was computed first using RANS. An LES was then carried out and the interface between RANS and LES was located at the position at which the rear slanting surface and the roof intersect. No turbulent fluctuations were applied at the inlet of the LES domain. Since this flow is an external flow in which the pressure field around the body is dependent on the flow in the entire region, it is questionable whether it is possible to decouple the RANS simulation and the LES simulation. A better approach - and also more expensive - would be to make the RANS and LES computations concurrently; a larger computational time step should probably be used in the RANS region to reduce the computational effort. Jarrin et al. ${ }^{5}$ used the SEM (Synthetic Eddy Method) method to impose turbulent fluctuations at the RANS-LES interface. They applied the method to channel flow, square duct flow and the flow over a trailing edge. Mary ${ }^{6}$ used zonal RANS-LES to predict the flow in an internal duct. Turbulent fluctuations from a database were used and re-scaled at the RANS-to-LES interface. This work also invoked an interface from LES to RANS at which the resolved turbulent fluctuations were dampened by means of a time filter. Zhang et al. ${ }^{7}$ used forcing at the interface between RANS and LES. The forcing was adjusted in order to match a prescribed Reynolds shear stress profile somewhere downstream of the RANS-LES interface. The approach was applied to channel flow and the flow around an airfoil. Forcing was also used in Ma et al., ${ }^{8}$ which was created using the SGS stress tensor from a scale-similarity model by selecting only the instantaneous SGS stress that contributes to backscatter. ${ }^{9}$ Shur et al. ${ }^{10}$ proposed a new recycling method in a interface zone between RANS and LES. They evaluated the method for for flat plate boundary flow and the flow over an two-dimensional airfoil.

In the present work, an embedded LES method is verified and applied to turbulent channel flow and a flow over a hump. The LES region is placed downstream of the upstream RANS region. In general, the LES region may be embedded in between upstream and downstream RANS regions. The emphasis in the present study is however on the RANS-LES coupling over the interface when going from an upstream RANS region to a downstream LES region as illustrated in Figs. 1 and 2(a). In this type of configuration, the critical issue in the RANS-LES coupling is how to create resolved turbulence at the interface and how to dampen modelled RANS turbulence when the flow enters into the LES region. The embedded LES method investigated in this work is based on the PANS (Partially Averaged-Navier-Stokes) modelling approach, ${ }^{11-13}$ which is a modified $k-\varepsilon$ model that can operate in either RANS mode or LES mode. An extension of PANS was recently proposed in which a four-equation $k-\varepsilon-\zeta-f$ model is used. ${ }^{14}$ In the present work, a 
low-Reynolds number (LRN) PANS model ${ }^{15}$ is used in both the RANS and the LES regions.

Interface

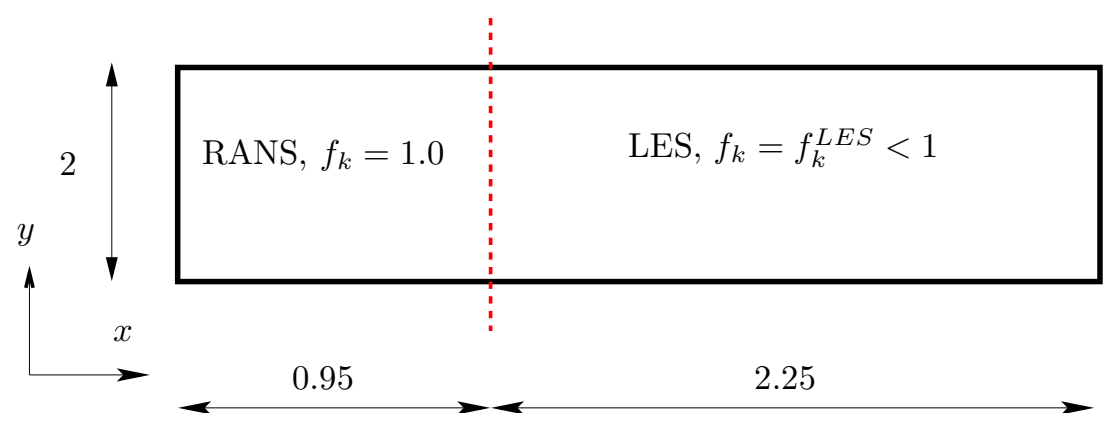

Figure 1. Channel flow configuration. The interface separates the RANS and the LES regions.

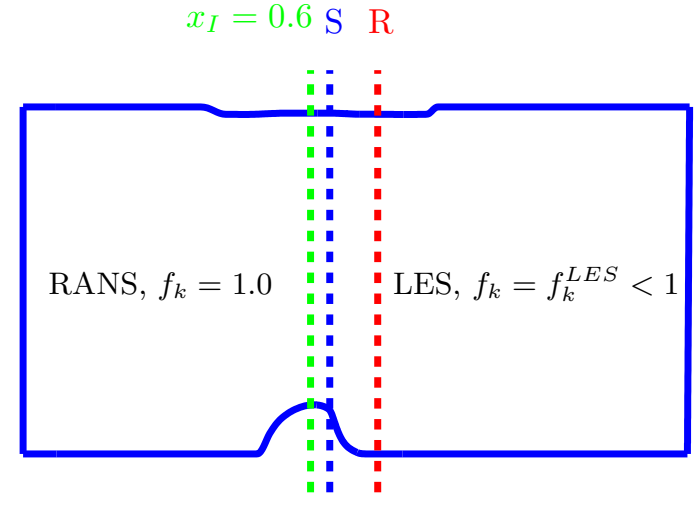

(a) Interface at $x=0.6$, Separation $x_{S}=0.65$; reattachment $x_{R}=1.1$. Not to scale.

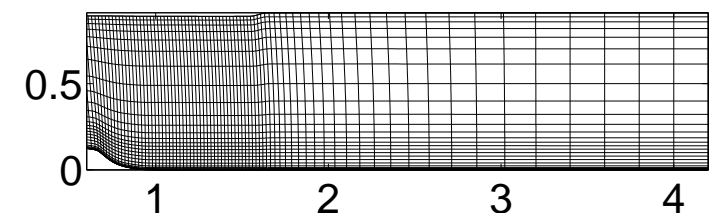

(b) LES Grid. Every $4^{\text {th }}$ grid line is shown.

Figure 2. Hump flow configuration and grid schematic.

The paper is organized as follows. The PANS turbulence model is briefly introduced and the embedded modelling approach is outlined in Section II. The method for generating turbulent synthetic inlet/interface fluctuations is then presented. Next, the numerical method is presented, followed by a presentation and discussion of the results in the subsequent section, and finally a summary and some concluding remarks are given.

\section{PANS-Based Embedded LES}

The PANS approach ${ }^{11-13}$ uses the ratio of modelled to total turbulent kinetic energy and the ratio of their dissipation rates, $f_{k}$ and $f_{\varepsilon}$, respectively. The partially-averaged governing equations for incompressible 
turbulent flows, invoking the PANS turbulent viscosity, $\nu_{u}$, reads

$$
\begin{gathered}
\frac{\partial \bar{u}_{i}}{\partial x_{i}}=0 \\
\frac{\partial \bar{u}_{i}}{\partial t}+\frac{\partial}{\partial x_{j}}\left(\bar{u}_{i} \bar{u}_{j}\right)=-\frac{1}{\rho} \frac{\partial \bar{p}}{\partial x_{i}}+\frac{\partial}{\partial x_{j}}\left[\left(\nu+\nu_{u}\right) \frac{\partial \bar{u}_{i}}{\partial x_{j}}\right]
\end{gathered}
$$

A recently developed LRN PANS model is employed, for improved modelling of near-wall turbulence, which reads $^{15}$

$$
\begin{aligned}
& \frac{\partial k_{u}}{\partial t}+\frac{\partial\left(k_{u} \bar{u}_{j}\right)}{\partial x_{j}}=\frac{\partial}{\partial x_{j}}\left[\left(\nu+\frac{\nu_{u}}{\sigma_{k u}}\right) \frac{\partial k_{u}}{\partial x_{j}}\right]+P_{u}-\varepsilon_{u} \\
& \frac{\partial \varepsilon_{u}}{\partial t}+\frac{\partial\left(\varepsilon_{u} \bar{u}_{j}\right)}{\partial x_{j}}=\frac{\partial}{\partial x_{j}}\left[\left(\nu+\frac{\nu_{u}}{\sigma_{\varepsilon u}}\right) \frac{\partial \varepsilon_{u}}{\partial x_{j}}\right]+C_{\varepsilon 1} P_{u} \frac{\varepsilon_{u}}{k_{u}}-C_{\varepsilon 2}^{*} \frac{\varepsilon_{u}^{2}}{k_{u}} \\
& \nu_{u}=C_{\mu} f_{\mu} \frac{k_{u}^{2}}{\varepsilon_{u}}, C_{\varepsilon 2}^{*}=C_{\varepsilon 1}+\frac{f_{k}}{f_{\varepsilon}}\left(C_{\varepsilon 2} f_{2}-C_{\varepsilon 1}\right), \sigma_{k u} \equiv \sigma_{k} \frac{f_{k}^{2}}{f_{\varepsilon}}, \sigma_{\varepsilon u} \equiv \sigma_{\varepsilon} \frac{f_{k}^{2}}{f_{\varepsilon}}
\end{aligned}
$$

The modification introduced by the PANS modelling as compared to its parent RANS model is highlighted by boxes. The model constants take the same values as in the LRN base model, ${ }^{16}$ i.e.,

$$
C_{\varepsilon 1}=1.5, C_{\varepsilon 2}=1.9, \sigma_{k}=1.4, \sigma_{\varepsilon}=1.4, C_{\mu}=0.09
$$

The model coefficient $f_{\varepsilon}$ is set to one, and for the baseline model $f_{k}=0.4$. The sensitivity to different values of $f_{k}$ is investigated. The damping functions, $f_{2}$ and $f_{\mu}$, have the forms, respectively, of

$$
\begin{aligned}
& f_{2}=\left[1-\exp \left(-\frac{y^{*}}{3.1}\right)\right]^{2}\left\{1-0.3 \exp \left[-\left(\frac{R_{t}}{6.5}\right)^{2}\right]\right\} \\
& f_{\mu}=\left[1-\exp \left(-\frac{y^{*}}{14}\right)\right]^{2}\left\{1+\frac{5}{R_{t}^{3 / 4}} \exp \left[-\left(\frac{R_{t}}{200}\right)^{2}\right]\right\}
\end{aligned}
$$

where $R_{t}=k_{u}^{2} /\left(\nu \varepsilon_{u}\right)$ and $y^{*}=\left(\varepsilon_{u} \nu\right)^{1 / 4} y / \nu$. At walls, $k_{u}=0$ is specified. For the dissipation rate, $\varepsilon_{u}$, the value at the adjacent wall nodes with a wall distance of $y$ is prescribed as

$$
\varepsilon_{u}=2 \nu \frac{k_{u}}{y^{2}}
$$

For the hump flow, it was found that this boundary condition for $\varepsilon_{u}$ gave numerical problems. Instead, $\varepsilon_{u}$ was computed as in the one-equation hybrid LES-RANS model ${ }^{9}$

$$
\varepsilon_{u}=\frac{k_{u}^{3 / 2}}{\ell}, \quad \ell=\kappa C_{\mu}^{-3 / 4} y\left[1-\exp \left(-0.2 k_{u}^{1 / 2} y / \nu\right)\right]
$$

with $\kappa=0.41$.

It may be noted that PANS is very similar to PITM ${ }^{17}$ (Partially Integrated Transport Model); also in PITM the $C_{\varepsilon 2}$ coefficient is reduced when going from steady RANS into turbulence resolving mode. 
In the present work, a constant value of $f_{k}$ is used in the LES domain. A value of $0.4^{15,18,19}$ has previously been found to be a suitable value. The reason why the model works well in LES mode on a reasonably fine mesh with $f_{k} \simeq 0.4$ seems to be that with this value both the $k_{u}$ and $\varepsilon_{u}$ equations are in local equilibrium, i.e. the production (source) and the destruction (sink) terms are in balance. ${ }^{18}$ Instantaneously, this is impossible because $C_{\varepsilon 1} \neq C_{\varepsilon 2}^{*}=1.11 C_{\varepsilon 1}$, but it turns out that in average both equations are in balance, i.e.

$$
C_{\varepsilon 1}\left\langle\frac{\varepsilon_{u}}{k_{u}} P_{u}\right\rangle=C_{\varepsilon 2}^{*}\left\langle\frac{\varepsilon_{u}^{2}}{k_{u}}\right\rangle
$$

and

$$
\left\langle P_{u}\right\rangle=\left\langle\varepsilon_{u}\right\rangle
$$

but

$$
C_{\varepsilon 1} \frac{\left\langle\varepsilon_{u}\right\rangle}{\left\langle k_{u}\right\rangle}\left\langle P_{u}\right\rangle \neq C_{\varepsilon 2}^{*} \frac{\left\langle\varepsilon_{u}\right\rangle^{2}}{\left\langle k_{u}\right\rangle}
$$

It is a general feature of any two turbulent quantities, $A$ and $B$, that $\langle A B\rangle\langle\langle A\rangle\langle B\rangle$ (Cauchy-Schwarz inequality). In the case of Eq. 7 , the correlation between $P_{u}, \varepsilon_{u}$ and $k_{u}^{-1}$ (left-hand side) is stronger than that between $\varepsilon_{u}^{2}$ and $k_{u}^{-1}$ (right-hand side). ${ }^{18}$ Hence, Eq. 7 is fulfilled although $C_{\varepsilon 2}^{*}>C_{\varepsilon 1}$. Since both $k_{u}$ and $\varepsilon_{u}$ equations are in local equilibrium on a fine mesh, the model acts as zero-equation model. When the grid is coarsened, the convection and diffusion will gradually play an role. Hence, there is no need to let $f_{k}$ vary; the $k_{u}$ and $\varepsilon_{u}$ are able to adapt from a well-resolved LES to a less well resolved LES without changing $f_{k}$. In RANS regions, we set, of course, $f_{k}=1$.

In embedded LES, RANS is used in the first part of the domain, from the inlet to a specified $x$ station denoted the interface. Figure 1 presents the flow configuration for channel flow in the first test case, where the interface is located at $x=0.95$. In the RANS region, $f_{k}$ in the LRN PANS model is set to one. At the interface, synthetic anisotropic fluctuations are introduced as additional source terms in the continuity and the momentum equations. In the LES region downstream of the interface, $f_{k}=f_{k}^{L E S}<1$. Baseline value of $f_{k}^{L E S}$ is, as discussed above, 0.4 .

All turbulence is modelled in the RANS region, since the PANS model returns to an LRN RANS model by setting $f_{k}=1.0$. Consequently, the modelled values of $k_{u}$ and $\nu_{u}$ are large. Downstream of the interface, they must be reduced to values corresponding to LES. This is achieved by setting the usual convection and diffusion fluxes of $k_{u}$ and $\varepsilon_{u}$ through the interface to zero. New "inlet" boundary conditions (i.e. interface 
conditions) are introduced via sources. It is found in the channel flow computations that the interface conditions of $k_{u}$ and $\varepsilon_{u}$ have a large effect on the resolved turbulence downstream of the interface. Indeed, this is the case also in general when prescribing turbulent (instantaneous) inlet boundary conditions in LES or DES, where a large value of turbulent viscosity usually dampens resolved turbulence.

The turbulent conditions for $k_{u}$ and $\varepsilon_{u}$ at the interface are set as follows.

- The modelled turbulent kinetic energy, $k_{i n t e r}$, is set from $k_{u}$ in the RANS region, $k_{R A N S}$, as

$$
k_{\text {inter }}=f_{k}^{L E S} k_{R A N S}
$$

where $k_{R A N S}$ is taken at $x=0.5$, see Fig. 1 .

- The modelled dissipation, $\varepsilon_{i n t e r}$, is set from $k_{\text {inter }}$ and an SGS length scale, $\ell_{s g s}$, which is estimated from the Smagorinsky model as

$$
\ell_{s g s}=C_{S} \Delta
$$

where $\Delta=V^{1 / 3}$ and $V$ is the volume of the cell. The modelled dissipation is then approximated from

$$
\varepsilon_{\text {inter }}=C_{\mu}^{3 / 4} k_{\text {inter }}^{3 / 2} / \ell_{\text {sgs }}
$$

The influence of different values of $C_{S}$ will be investigated. The expression for $\varepsilon_{\text {inter }}$ gives an increase in $\varepsilon$ over the interface (see Section V.A) which contributes to the decrease of the turbulent viscosity.

$k_{\text {inter }}$ and $\varepsilon_{\text {inter }}$ are transported by convection and diffusion from the RANS region into the LES region through the interface. The interface conditions reduce, as mentioned above, $k_{u}$ and $\nu_{u}$ across the interface. The modelled dissipation, $\varepsilon_{u}$, also decreases across the interface near the wall ( $y^{+} \lesssim 7$ for the channel flow), but further away from the wall it increases, see Section V.A; in this way it helps to decrease $\nu_{u}$ across the interface in the larger part of the boundary layer.

\section{Anisotropic Synthetic Turbulent Fluctuations}

Anisotropic synthetic fluctuations of velocity components ${ }^{20-22}$ are added at the interface plane. The turbulent fluctuations that are generated will be homogeneous. The method can be summarized by the following steps. 
1. A Reynolds stress tensor, $\left\langle u_{i}^{\prime} u_{j}^{\prime}\right\rangle$, is taken from DNS data for turbulent channel flow. Since the generated turbulence is homogeneous, it is sufficient to choose one location of the DNS data. In this work, the Reynolds stresses at $y^{+} \simeq 16$ of the DNS channel data at $R e_{\tau}=590,{ }^{23}$ where $\left\langle u^{\prime} u^{\prime}\right\rangle$ - and hence the degree of anisotropy - is largest, are used, which reads

$$
\left\langle u_{i}^{\prime} u_{j}^{\prime}\right\rangle=\left[\begin{array}{ccc}
7.67 & -0.662 & 0 \\
-0.662 & 0.32 & 0 \\
0 & 0 & 1.50
\end{array}\right]
$$

This is used for both the channel and the hump test case.

2. The principal directions, $\eta_{i}$, are computed for the $\left\langle u_{i}^{\prime} u_{j}^{\prime}\right\rangle$ tensor.

3. Isotropic synthetic fluctuations, $u_{i, i s o}^{\prime}$, are then generated in the principal directions of $\left\langle u_{i}^{\prime} u_{j}^{\prime}\right\rangle$. The code for generating the isotropic fluctuations can be downloaded. ${ }^{24}$

4. The isotropic synthetic fluctuations in the $\eta_{i}$ directions are multiplied by the eigenvalues of $\left\langle u_{i}^{\prime} u_{j}^{\prime}\right\rangle$ giving a new field of fluctuations, $v_{i}^{\prime}$, so that $\left\langle v_{1}^{\prime} v_{1}^{\prime}\right\rangle \neq\left\langle v_{2}^{\prime} v_{2}^{\prime}\right\rangle$. Note that $v_{1}^{\prime}$ and $v_{2}^{\prime}$ are still uncorrelated, i.e. $\left\langle v_{1}^{\prime} v_{2}^{\prime}\right\rangle=0$.

5. The $v_{i}^{\prime}$ fluctuations are transformed to the computational coordinate system, $x_{i}$; these anisotropic fluctuations are denoted $u_{i, a n i s o}^{\prime}$. The Reynolds stress tensor of the synthetic anisotropic fluctuations is now identical to the DNS Reynolds stress tensor, i.e. $\left\langle u_{i, a n i s o}^{\prime} u_{j, a n i s o}^{\prime}\right\rangle=\left\langle u_{i}^{\prime} u_{j}^{\prime}\right\rangle$

6. Since the $u_{i, \text { aniso }}^{\prime}$ are homogeneous, the Reynolds stresses, $\left\langle u_{i, \text { aniso }}^{\prime} u_{j, \text { aniso }}^{\prime}\right\rangle$, have constant values in the inlet plane. However, the fluctuations are dampened near the wall so as to reach a value of zero on the wall surface. For the hump flow, the fluctuations are also dampened in the bulk flow, see Section V.B.

7. In the channel flow, the Reynolds shear stress changes sign across the centerline. Hence, the sign of $u_{2, \text { aniso }}^{\prime}$ is changed in the upper half $(y>1)$ of the channel.

8. The correlation in time is achieved by an asymmetric time filter ${ }^{25}$ (shown only for the streamwise fluctuation here)

$$
\left(\mathcal{U}^{\prime}\right)^{m}=a\left(\mathcal{U}^{\prime}\right)^{m-1}+b\left(u_{\text {aniso }}^{\prime}\right)^{m}
$$




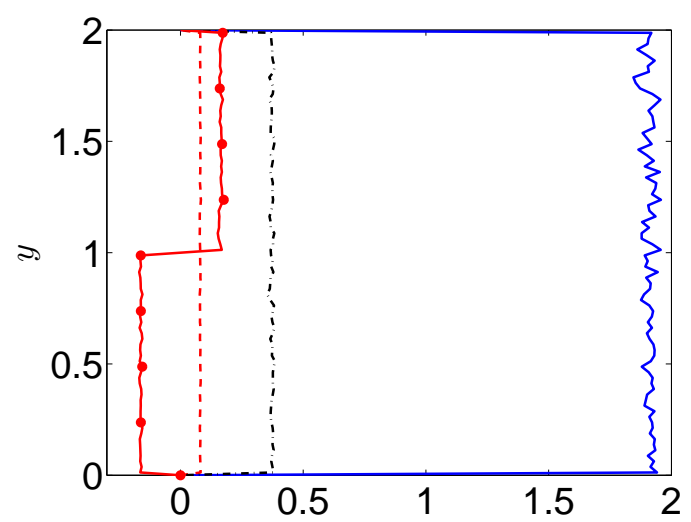

(a) —— : $u_{r m s}^{2} / u_{\tau}^{2} ;---: v_{r m s}^{2} / u_{\tau}^{2} ;-\cdot-: w_{r m s}^{2} / u_{\tau}^{2}$; $\multimap:\left\langle u^{\prime} v^{\prime}\right\rangle / u_{\tau}^{2}$.

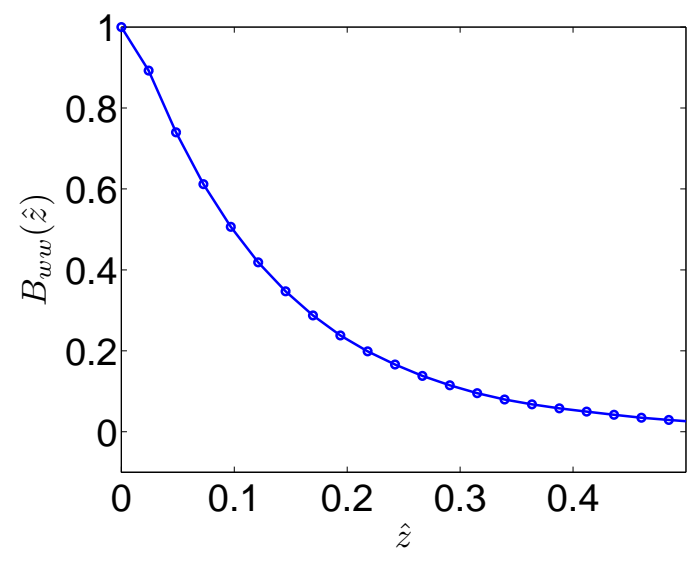

(b) Two-point correlation.

Figure 3. Channel flow - prescribed synthetic Reynolds stresses at the RANS-LES interface.

where $m$ is the current time step and $a=0.954, b=\left(1-a^{2}\right)^{1 / 2}$. Constant $a$ is related to the integral time scale, $\mathcal{T}$, as

$$
a=\exp (-\Delta t / \mathcal{T})
$$

where $\Delta t$ is the computational time step. Constant $b$ is given by the requirement that $\left\langle\mathcal{U}^{\prime 2}\right\rangle=\left\langle u_{\text {aniso }}^{\prime 2}\right\rangle$.

Figures 3 and 4 present the Reynolds stresses of the synthetic fluctuations for the channel flow (interface fluctuations at $x=0.95$ ) and the hump flow (inlet fluctuations at $x=0.6$ ), respectively. As can be seen, they are constant (homogeneous) across the boundary layer except close to the walls, where they are dampened linearly to zero. For the hump flow, they are also dampened in the freestream region, see Section V.B. Note that the shear stress changes sign at the center of the channel as it should. Furthermore, it is constant in the upper and lower half of the channel which is a consequence of the assumption of homogeneity. The fluctuations could be scaled with, for example, a $k$ profile from experiments, RANS, or DNS. The main argument for not doing this is that the prescribed integral length scale (computed from the two-point correlation) in the $y$ direction would then be modified. Furthermore, it was found in a previous work ${ }^{25}$ that a re-scaling actually gives poorer predictions. 


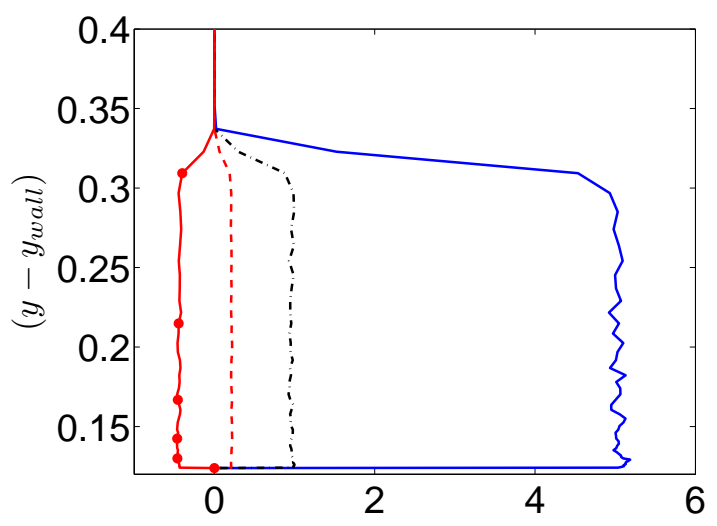

(a) —— : $u_{r m s}^{2} / u_{\tau}^{2} ;---: v_{r m s}^{2} / u_{\tau}^{2} ;-\cdot-: w_{r m s}^{2} / u_{\tau}^{2}$; $\multimap:\left\langle u^{\prime} v^{\prime}\right\rangle / u_{\tau}^{2}$.

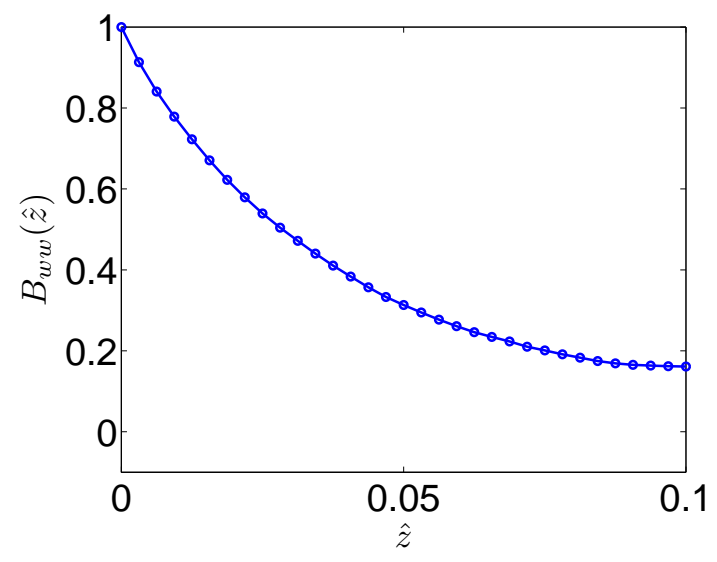

(b) Two-point correlation.

Figure 4. Hump flow - prescribed synthetic Reynolds stresses at the inlet.

\section{Numerical Method}

An incompressible, finite volume code is used in all the computations. ${ }^{26}$ The numerical procedure is based on an implicit, fractional step technique with a multigrid pressure Poisson solver ${ }^{27}$ and a non-staggered grid arrangement. For the momentum equations in the LES region (downstream of the interface), central differencing is used for the channel flow. For the hump flow, central differencing is blended with $5 \%$ upwinding using the bounded second-order upwind scheme of van Leer. ${ }^{28}$ The upwinding is used because in Ref. 29 it was found that pure central differencing gave rise to unphysical resolved stresses near the inlet. The Crank-Nicolson scheme is used in the time domain but for the pressure gradient term it was found to be unstable for the hump flow and hence a fully implicit scheme is used for this term.

To prevent the imposed synthetic turbulent fluctuations at the interface from propagating upstream in the channel flow, a dissipative discretization scheme is used in the RANS region upstream of the interface. We use here a bounded second-order upwind van Leer scheme ${ }^{28}$ in space, and the Crank-Nicolson scheme (except for the pressure gradient) in the time domain.

A hybrid central/upwind (first order) ${ }^{30}$ scheme in space and the Crank-Nicolson scheme for time discretization are used when solving for the $k_{u}$ and $\varepsilon_{u}$ equations in the entire domain. 


\section{Results and Discussion}

\section{V.A. Channel Flow}

The Reynolds number for the channel flow is $R e_{\tau}=950$ based on the friction velocity, $u_{\tau}$, and half the channel width, $\delta$. In the present simulations, we have normalized such that $\rho=1, \delta=1$ and $u_{\tau} \simeq 1$, see Fig. 1. The relatively low Reynolds number $\left(R e_{\tau}=950\right)$ is chosen to allow an accurate simulation of the flow when the PANS model is used in LES mode. With a $3.2 \times 2 \times 1.6$ domain, a mesh with $64 \times 80 \times 64$ cells is used in, respectively, the streamwise $(x)$, the wall-normal $(y)$ and the spanwise $(z)$ direction, see Fig. 1. The resolution is approximately (the wall shear stress varies slightly along the wall) $[48,24](x, z)$ in viscous units; in the $y$ direction, minimum (near the wall) and maximum (in the center) cell side are 0.6 and 10 , respectively. The inlet mean velocities are set as $V_{i n}=W_{i n}=0$ and $^{31}$

$$
U_{i n}^{+}= \begin{cases}y^{+} & y^{+} \leq 5 \\ -3.05+5 \ln \left(y^{+}\right) & 5<y^{+}<30 \\ \frac{1}{0.4} \ln \left(y^{+}\right)+5.2 & y^{+} \geq 30\end{cases}
$$

The inlet $k_{u}$ and $\varepsilon_{u}$ conditions are created by computing fully developed channel flow with the LRN PANS model in RANS mode (i.e. with $f_{k}=1$ ). The reason why the inlet velocity is taken from Eq. 15 rather than from LRN PANS $\left(f_{k}=1\right)$ is that the latter does not perfectly match the log-law.

Convective boundary conditions are used at the outflow and periodic conditions are employed in the spanwise direction. The anisotropic synthetic fluctuations described in Section III are added at the interface. The spanwise integral length scale calculated from the two-point correlation, see Fig. 3(b), of the $w_{\text {aniso }}^{\prime}$ fluctuations is 0.13 . The resulting integral time scale of the synthetic fluctuations is 0.015 (the numerical time step is 0.000625$)$. The interface condition for $\varepsilon_{u}$ is computed with the baseline value $C_{S}=0.07$, see Eq. 11, and the interface condition for $k_{u}$ is computed from Eq. 10.

Figure 5 presents the mean velocity and the resolved and modelled shear stresses at three streamwise locations, $x=0.19,1.25$ and 3 (recall that the interface is located at $x=0.95$ ). The sign of the modelled stresses is reversed in the figure in order to enhance readability; furthermore the modelled stresses are shown only in the lower half of the channel. At $x=3$, the predicted velocity agrees very well with the log-law. This suggests that the modelled turbulent shear stresses have been effectively adapted and are appropriate 
to enable a very good distribution of the mean flow. The resolved shear stress is - as expected - zero at the first location, which is located in the RANS region; the modelled shear stresses is large. At $x=1.25$ (i.e. $0.3 \delta$ downstream the interface), the resolved shear stress resembles the prescribed shear stress at the interface, but its form is reasonably close to a fully developed profile at $x=3(2.05 \delta$ downstream the interface). With a further extended channel length, it is believed that the resolved turbulence would be further re-established and the resolved turbulent shear stress should be well recovered correspondingly. The modelled shear stresses are negligible downstream of the interface.
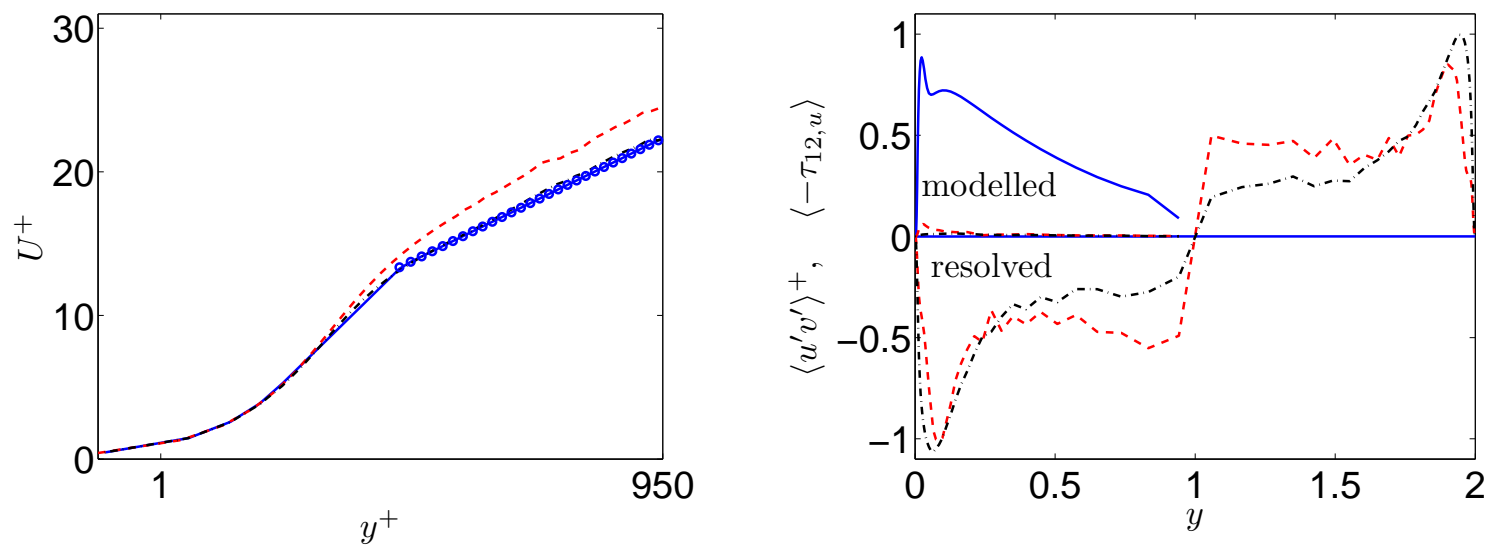

Figure 5. Channel flow. Time-averaged streamwise $(U)$ velocity and resolved and modelled (opposite sign) shear stresses. $-x=0.19 ;---x=1.25 ;--x=3$. The log-law is plotted in symbols.

The RMS of resolved velocity fluctuations, $u_{r m s}, v_{r m s}$ and $w_{r m s}$, at $x=3$, the peak values of $u_{r m s}$ and the turbulent viscosity versus streamwise position are presented in Fig. 6. The near-wall distribution of the RMS of velocity fluctuations is in reasonable agreement with DNS data $\left(u_{r m s}\right.$ is somewhat too large). They are, however, over-predicted in the center region. This has been caused by the homogeneous fluctuation profiles imposed at the interface. The resolved streamwise velocity fluctuations are zero in the RANS region as they should, as shown in Fig. 6(b), of which the maximum RMS values increase sharply over the interface thanks to the imposed fluctuations. The turbulent viscosity is reduced at the interface from its peak RANS value of approximately 80 to a value relevant for LES with $\nu_{u, p e a k} / \nu \simeq 1$.

Figures 7 and 8 present the sensitivity to the prescribed inlet turbulent length scale, i.e. to $C_{S}$ in Eq. (11). An increased length scale (equivalent to a reduced $\varepsilon_{u}$ ) at the interface gives, as expected, an increased turbulent viscosity and a reduced peak of the resolved turbulent shear stress in the LES region. The baseline value, $C_{S}=0.07$, gives the best results. The friction velocity, shown in Fig. 8(b), quickly 


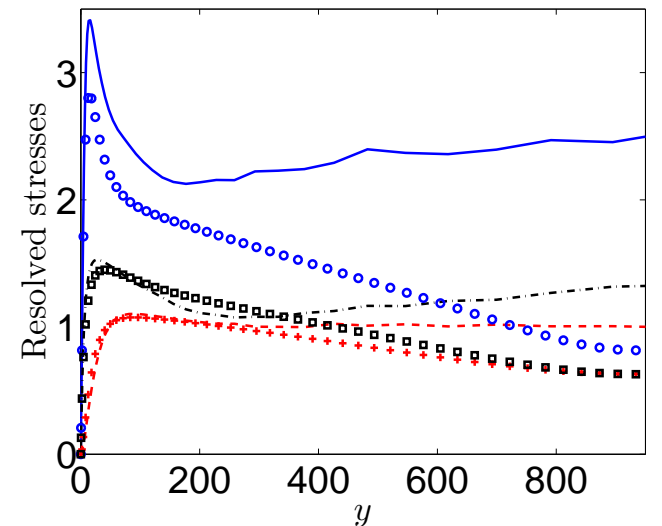

(a) Resolved fluctuations as $x=3$. $u_{r m s}^{+}$; $--v_{r m s}^{+} ;---w_{r m s}^{+} ;$markers: DNS data. ${ }^{32}$

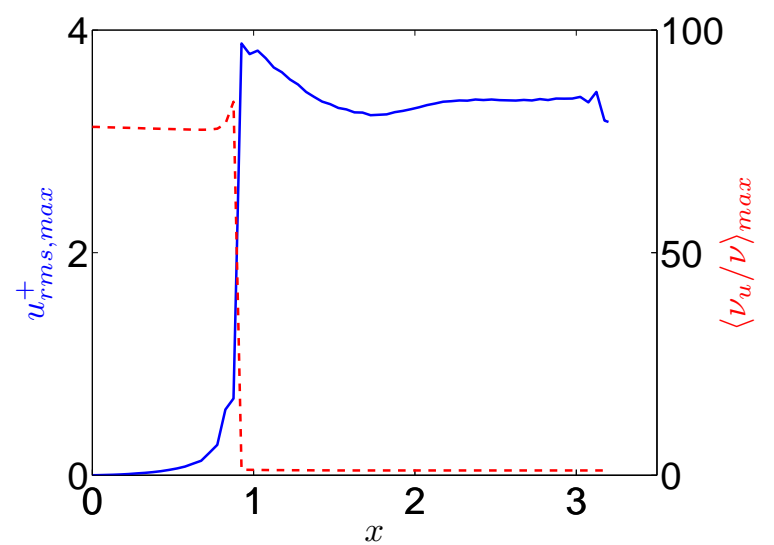

(b) - - : Turbulent viscosity (right $y$ axis); — : maximum $\left\langle u^{\prime} u^{\prime}\right\rangle^{+}$(left $y$ axis)

Figure 6. Channel flow. (a) Resolved normal resolved RMS fluctuations at $x=3$; (b) Maximum $u_{r m s}^{+}$and $\left\langle\nu_{u} / \nu\right\rangle$ versus

approaches the fully developed value of $u_{\tau}=1$ with $C_{S}=0.07$, whereas larger values of $C_{S}$ delay the re-establishment of $u_{\tau}$ towards $u_{\tau}=1$, which instead approaches a smaller value. The reason is that the turbulent viscosity becomes so large that it tends to dampen the resolved fluctuations. If the channel were long enough, the resolved turbulence would probably be fully dampened because of too large turbulent viscosities. It is noted that, for $C_{S}=0.07, u_{\tau}$ exhibits oscillations in the LES region due to the use of central differencing scheme. The same behavior was observed in previous simulations. ${ }^{25}$ Nonetheless, this behavior never appears in simulations of a fully developed channel flow in which the oscillations are automatically suppressed by the periodic streamwise boundary conditions. Away from the wall the numerical oscillations are not visible; the resolved, turbulent fluctuations are orders of magnitudes larger (compare the oscillations in Fig. 6(a)). Note that for for $C_{S}=0.1$ and $C_{S}=0.2$, the $u_{\tau}$-distributions are shown for every second node, and hence no oscillations are presented in Fig. 8(b).

With the baseline value $C_{s}=0.07$, the friction velocity in Fig. 8 approaches the target value of one over a distance of less than two $\delta$ downstream of the interface. This is considerably better than what was achieved with the SEM method ${ }^{5}$ which required $10 \delta$ to recover the target value for the skin friction. Keating et $a l .{ }^{33}$ used synthetic fluctuations for inlet boundary conditions and their skin friction was restored much later $(\simeq 10 \delta)$ than in the present work. Adamian and Travin ${ }^{34}$ proposed a modified SEM method which they used for inlet fluctuations in channel flow simulations, and showed that the skin friction was restored 
to the target value within $\delta<x<2 \delta$.

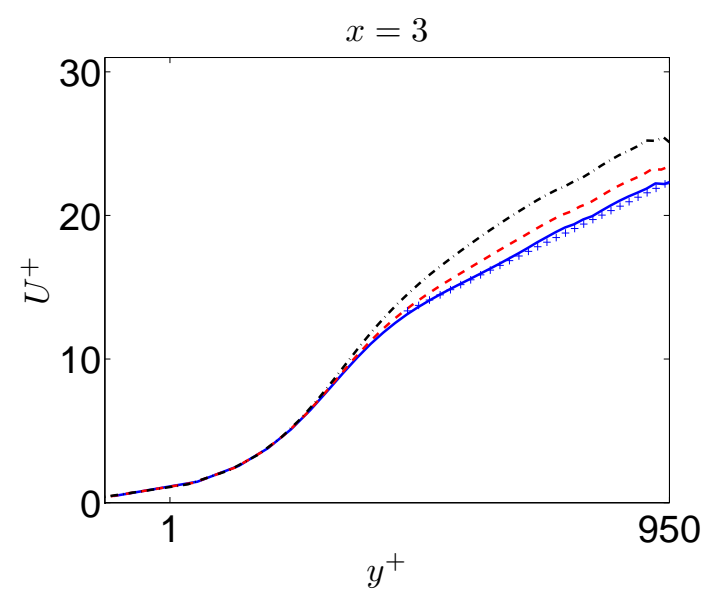

(a) Mean velocity

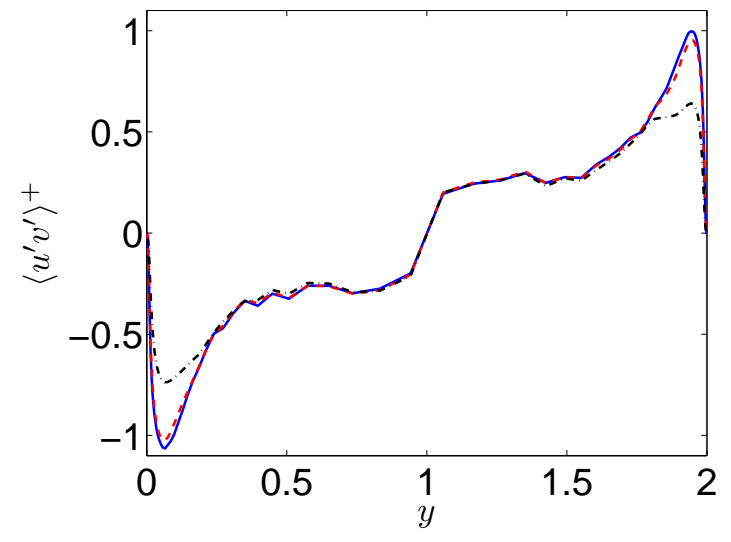

(b) Resolved shear stress

Figure 7. Channel flow. Sensitivity to $C_{S}$ when prescribing interface $\varepsilon_{u}$ condition using Eq. 11. (a) Mean velocity at $x=3$; (b) Resolved shear stresses at $x=3$. _ : $C_{S}=0.07$; - - : $C_{S}=0.1$; - - : $C_{S}=0.2$.

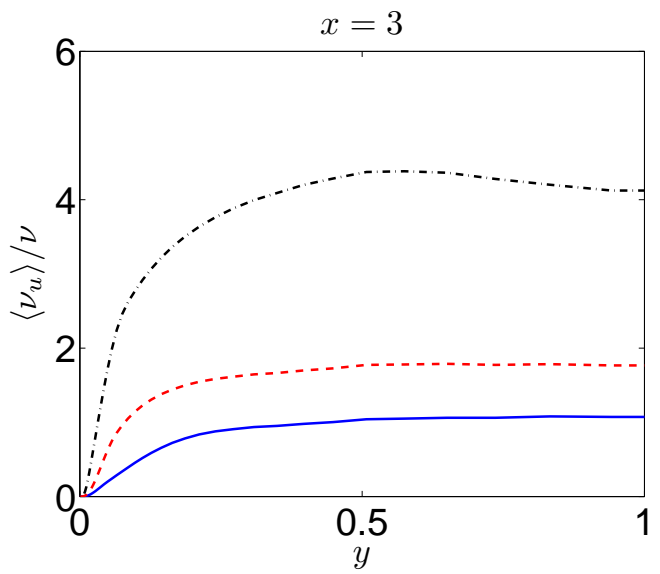

(a) Turbulent viscosity

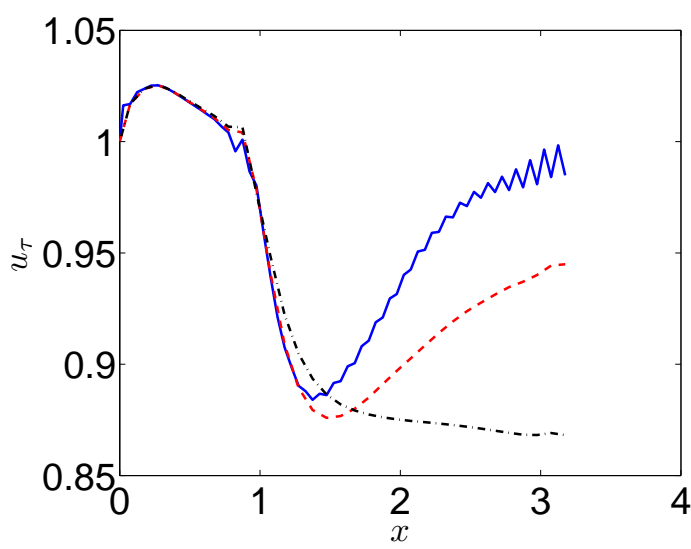

(b) Friction velocity

Figure 8. Channel flow. Sensitivity to $C_{S}$ when prescribing interface $\varepsilon_{u}$ condition using Eq. 11. (a) Turbulent viscosity at $x=3$; (b) Development of friction velocity in the streamwise direction. - : $C_{S}=0.07$; - - : $C_{S}=0.1$; - - : $C_{S}=0.2$.

Figures 9 and 10 present predictions at $x=3$ using different $f_{k}$ values in the LES region. It is shown that both $f_{k}=0.2$ and $f_{k}=0.4$ give very good results and that $f_{k}=0.6$ generates somewhat too a large modelled eddy viscosity, resulting in a small over-prediction of $U^{+}$. Although not shown here, it is noted that $f_{k}=0.3$ and 0.5 produce almost as good results as the baseline value of $f_{k}=0.4$.

Figure 11 compares the modelled dissipation, $\varepsilon_{u}$, in the RANS region upstream of the interface with $\varepsilon_{\text {inter }}$, see Eq. 12. The latter is, as can be seen, larger than $\varepsilon$ in the RANS region (except close to the wall, 


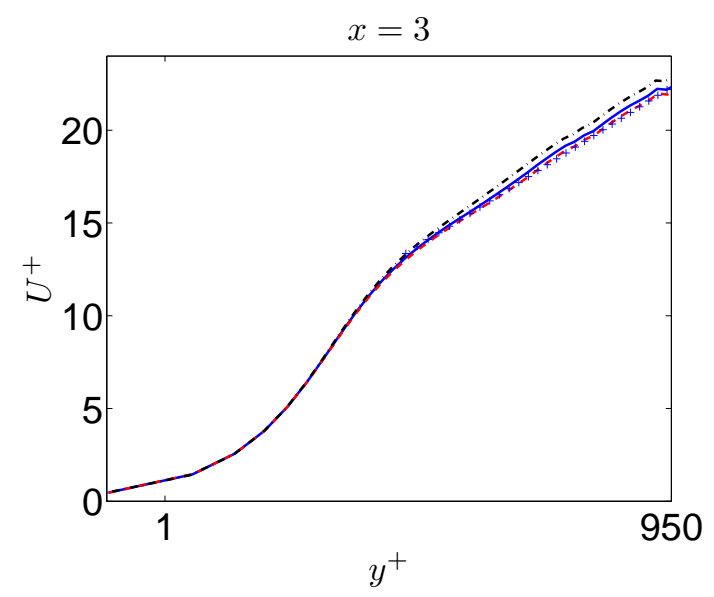

(a) Mean velocity

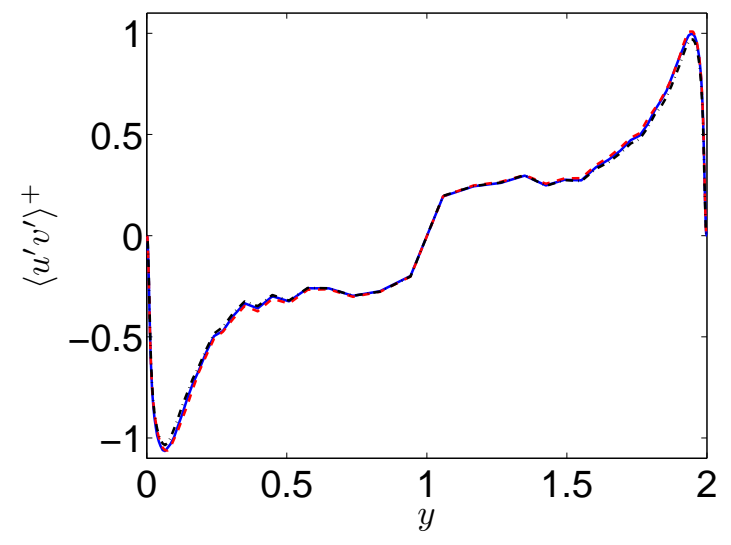

(b) Resolved shear stress

Figure 9. Channel flow. Sensitivity to $f_{k}$ in the LES region. Mean velocity and resolved shear stresses at $x=3$. $f_{k}=0.4 ;---: f_{k}=0.2 ;-\cdot-f_{k}=0.6$.

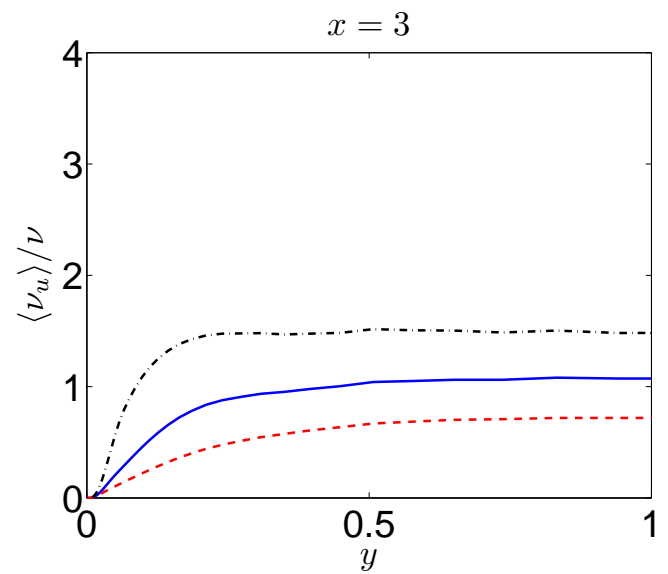

(a) Turbulent viscosity

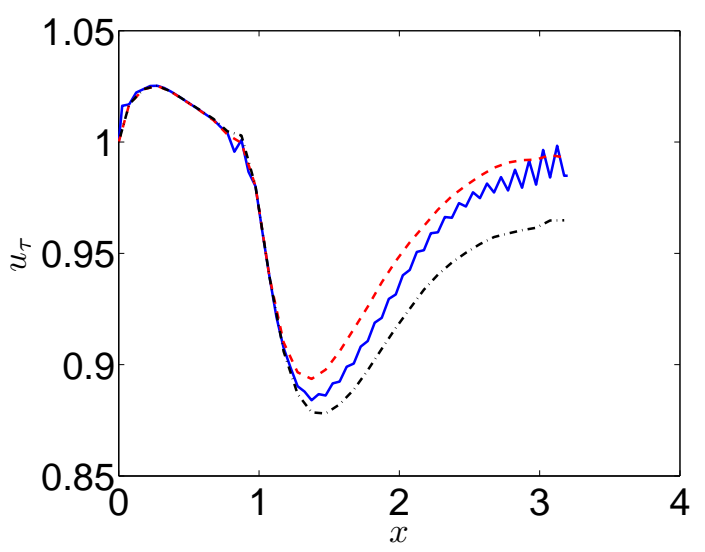

(b) Friction velocity

Figure 10. Channel flow. Sensitivity to $f_{k}$ in the LES region. (a) Turbulent viscosity at $x=3$; (b) Development of friction velocity in the streamwise direction. _— $: f_{k}=0.4 ;---: f_{k}=0.2 ;--f_{k}=0.6$.

$\left.y^{+}<7\right)$. Hence the interface condition for $\varepsilon$, Eq. 12, increases $\varepsilon$ across the interface and thereby decreasing $\nu_{u}$. This means that both the interface condition on $k_{u}$ (Eq. 10) and on $\varepsilon_{u}$ contribute to the reduction of $\nu_{u}$ across the interface.

Figures 12 present simulations obtained on a coarse spanwise resolution, $N_{k}=32$, using the baseline PANS model $\left(f_{k}=0.4\right.$ and $\left.C_{s}=0.07\right)$. It should be kept in mind that, in fully developed channel flow, the coarse resolution does not give good agreement with DNS and the log-law. Hence the velocity profiles from a fully developed channel flow is included in Figs. 12 The interface conditions trigger the flow into resolved mode in a similar way as for the fine resolution, but the development towards fully developed conditions is 


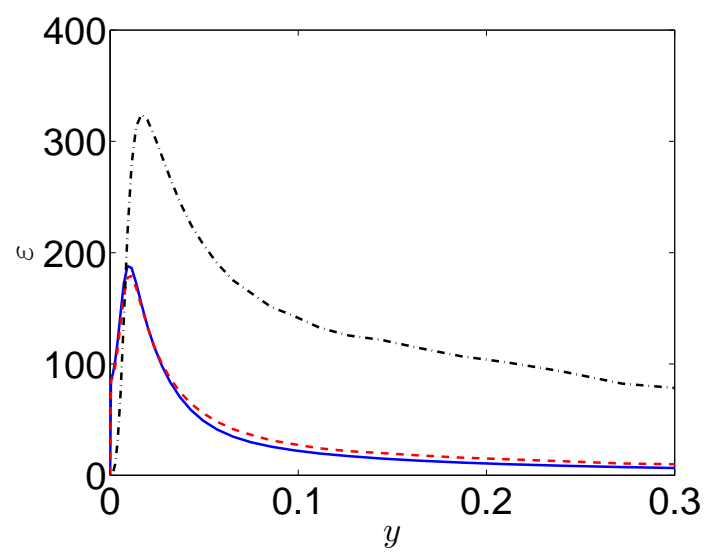

Figure 11. Channel flow. Dissipation. — $: \varepsilon$ at $x=0.5 ;---: \varepsilon$ immediately upstream the interface at $x=0.9---$ : $\varepsilon_{\text {inter }}$ (see Eq. 12) with $C_{s}=0.07$.

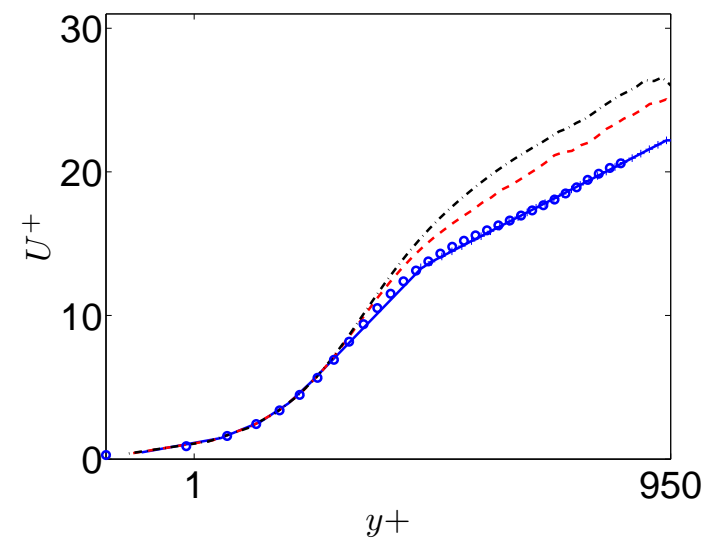

(a) Mean velocity

Figure 12. Channel flow. Coarse grid resolution in spanwise direction, $N_{k}=32 . \quad f_{k}=0.4, C_{S}=0.07 .-x=0.19$; $---x=1.25 ;--x=3$; ○: DNS data; ${ }^{32}+$ : fully developed channel flow.

somewhat slower for the coarse resolution compared to the fine resolution (not shown).

\section{V.B. Hump flow}

The hump flow has been studied previously using LES ${ }^{35,36}$ and DES. ${ }^{36}$ Wall functions were used in Ref. 35, whereas the near-wall flow was resolved in Ref. 36 with a refined mesh in the wall-normal directions. This flow was also studied by the present authors in Ref. 29. The main difference in the present study is that 95\% central differcing and 5\% upwinding are used for discretizing the convection term in the momentum equations whereas in Ref. 29 100\% central differencing was used. The upwinding is used to suppress the unphysical oscillations in the resolved stresses which were seen near the inlet in Ref. 29. 
The Reynolds number of the hump flow is $R e_{c}=936000$, based on the hump length, $c$, and the inlet mean velocity at the centerline, $U_{i n, c}$. In the present simulations, the value of $\rho, c$ and $U_{i n, c}$ have been set to unity by adapting the molecular viscosity to have the Reynolds number specified. The configuration is given in Fig. 2(a). Experiments were conducted by Greenblatt. ${ }^{37,38}$ The maximum height of the hump, $h$, and the channel height, $H$, are given by $H / c=0.91$ and $h / c=0.128$, respectively. The baseline mesh has $312 \times 120 \times 64$ cells with $Z_{\max }=0.2$. The grid was created by the group of Prof. Strelets in St. Petersburg and is the mandatory grid in the ATAAC project (see Acknowledgments). The $x-y$ grid is shown in Fig. 2(b).

There are side-wall effects (3D flow) near the side plates in the experiment. Hence, in order to compensate for the blockage effect of the side plates in the computation, the surface shape of the upper wall (above the hump) is modified and the upper wall is moved slightly downwards, see Fig. 2(a). The ratio of the local cross-sectional area of the side plates (facing the flow) to the cross sectional area of the tunnel enclosed by the side plates was computed. This ratio was used to scale the local height of the channel and thus modifying the contour shape of the upper wall.

Neumann conditions are used at the outflow section located at $x=4.2$. Slip conditions are used at the upper wall and symmetric boundary conditions are used on the spanwise boundaries. Inflow boundary (at $x=0.6$ ) conditions are taken from 2D RANS SST $k-\omega$ simulations carried out by the group of Prof. Strelets in St. Petersburg. The distributions of $U$ and $V$ at $x=0.6$ from the RANS simulation are used together with $W=0$ as mean inlet velocities to which the fluctuating velocity $\mathcal{U}^{\prime}, \mathcal{V}^{\prime}$ and $\mathcal{W}^{\prime}$, obtained with Eq. 13 , are superimposed. The computed integral length scale for the synthetic inlet fluctuations is $\mathcal{L} \simeq 0.040$ (see the two-point correlation in Fig. 4(b)) and the integral time scale $\mathcal{T} \simeq 0.038$. It is noted that the prescribed inlet integral length scale is rather large (approximately equal to the inflow boundary layer thickness). The reason is that synthetic fluctuations with a large integral length scale are efficient in generating resolved turbulent fluctuations. ${ }^{25}$

The inlet fluctuations, $\mathcal{U}^{\prime}, \mathcal{V}^{\prime}$ and $\mathcal{W}^{\prime}$, are reduced to zero in the off-wall region by multiplication of the blending function $f_{b l}$

$$
f_{b l}=\max \left\{0.5\left[1-\tanh \left(\frac{y-y_{b l}-y_{w a l l}}{B}\right)\right], D\right\}, \quad y_{b l}=0.2, \quad B=0.01
$$

This makes the fluctuations go to zero at the distance of $y_{b l} \simeq 0.2$ from the wall over the distance $B=0.01$, 
see Fig. 4. The freestream turbulence is determined by $D$, which takes a value of $D=0.02$. The inlet boundary condition for $\varepsilon_{u}$ is computed with the baseline value $C_{S}=0.1$, see Eq. 11 , and the inlet condition for $k_{u}$ is computed from Eq. 10 .

The time step is set to $\Delta t=0.002$. Before averaging is started, 7500 time steps are run and sampling is then done for another 7500 time steps. The entire CPU time on one Intel i5-2400 core under Linux is approximately 75 hours.

Figures 13-17 present results obtained with different magnitudes of the synthetic turbulent inlet fluctuations. Three different magnitudes are used: the baseline value (see Fig. 4(a)), and 50\% larger and $50 \%$ smaller, respectively, than the baseline value. All three predictions give fairly good agreement with experiments. It is shown that the larger the inlet fluctuations, the stronger the recirculation on the lee side of the hump. Large inlet fluctuations create a larger turbulent resolved diffusion of the free shear layer above the recirculation bubble which induces a more intensive backflow as shown in Figs. 13(b) and $14(x=0.8)$. This gives an earlier reattachment on the bottom wall after the hump. Moreover, as illustrated in Figure 16, large inlet fluctuations produce large resolved shear stresses in the backflow region. They are much larger than the experimental values. The smallest inlet fluctuations yield resolved shear stresses that are in much better agreement with the experiments, although they exhibit a much stronger increases from $x=0.65$ to $x=0.8$ than do the experimental shear stresses. This may have been caused by a poor resolution of the initial shear layer. The wall pressures (Fig. 13(a)) downstream the reattachment $(x>1.1)$ are consistent with the strength of recirculation: the stronger the recirculation and the earlier the reattachment, the earlier the pressure recovery.

The distributions of mean streamwise velocity plotted at different stations, as shown in Fig. 14, correspond well to the distribution of $C_{f}$ in Fig. 13(b). Except for the backflow in the recirculation bubble, the difference in the predicted mean flow is only marginal between the baseline case and the case with small inlet fluctuations. The case with large fluctuations differ rather much from the other two predictions: the recirculation in the former case is too strong and the predicted recovery rate is somewhat too slow. It should be noted that measured velocities using two different experiment techniques have been used. For $x<1$, data from 2D PIV are used and for $x \geq 1$ we compared with 3D PIV measurements which have been spanwise averaged over an extension of $\Delta z=0.14$. For $x \geq 1$, there are data using both techniques and they are 

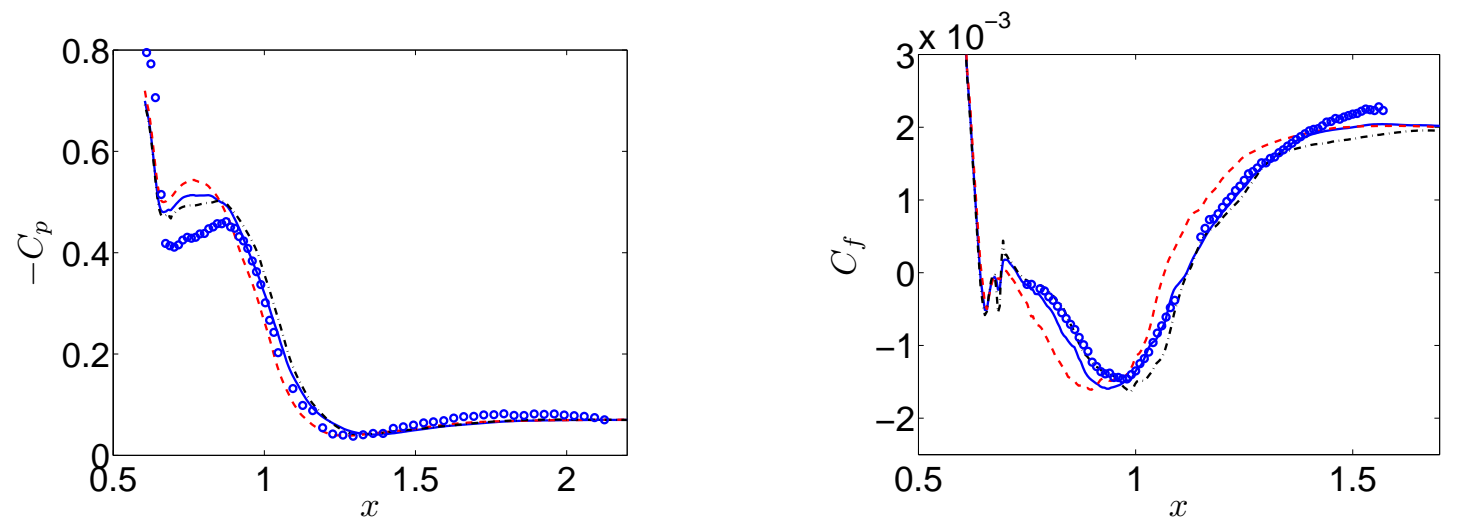

Figure 13. Hump Flow. (a) Pressure coefficient; (b) Skin friction.

- baseline inlet fluctuations (see Fig. 4); - - : $1.5 \times$ (baseline inlet fluctuations); - - : $0.5 \times$ (baseline inlet fluctuations); $\circ$ : experiments.
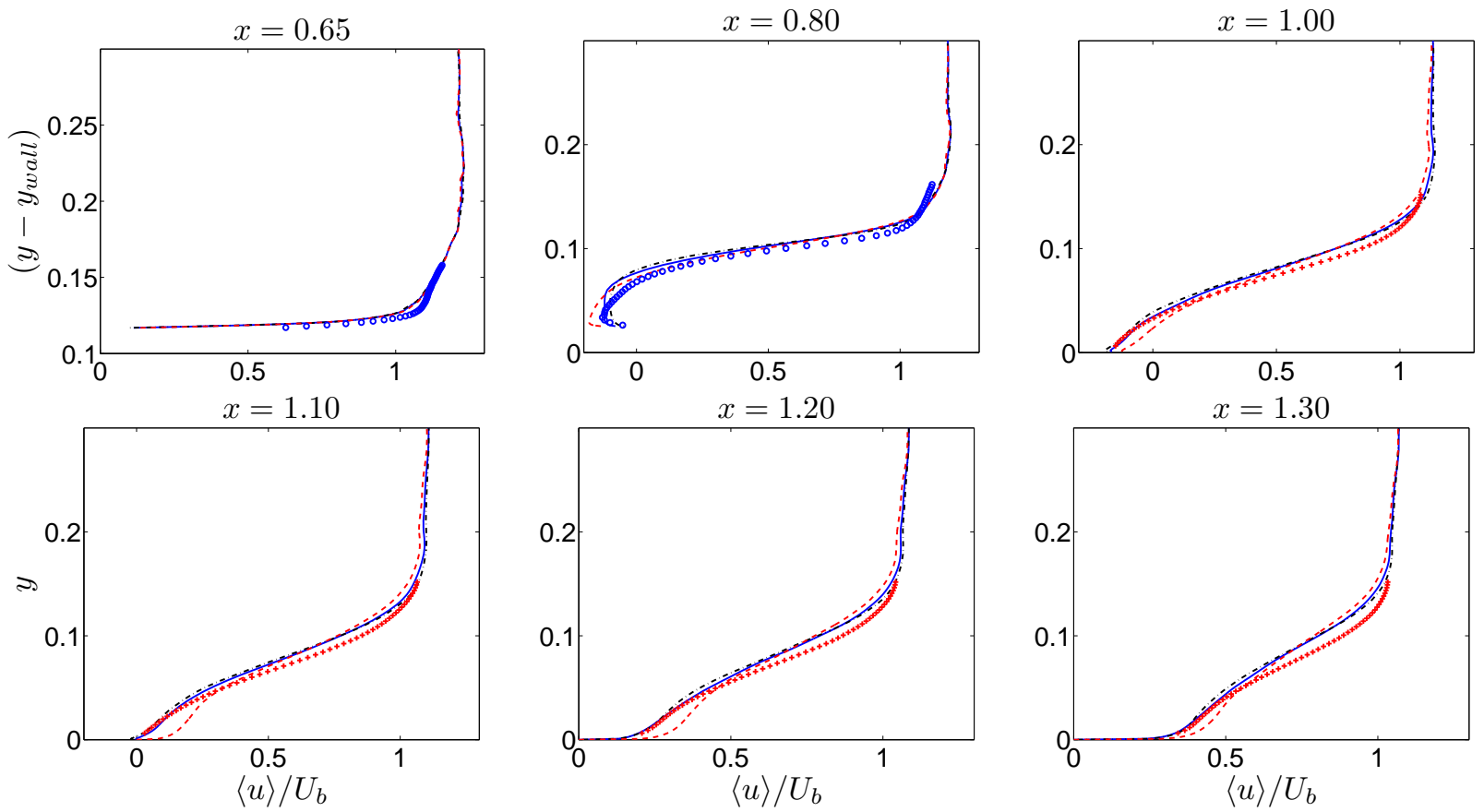

Figure 14. Hump flow: Mean velocity, $\langle\bar{u}\rangle \_$: baseline inlet fluctuations (see Fig. 4); - - : 1.5 $\times$(baseline inlet fluctuations); - - : $0.5 \times$ (baseline inlet fluctuations); ○: 2D PIV experiments; +: 3D PIV experiments.

compared in Fig. 15 with the baseline predictions; as can be seen both the predicted velocity and the 3D PIV data in the outer region decrease for increasing $x$. However, the 2D PIV velocity profiles in the outer region actually stay constant when moving from $x=1.10$ to $x=1.30$ whereas the velocity in the inner region increases. It seems that mass conservation is not satisfied in the 2D PIV data; or it may be that for $x>1.1$ the velocity decreases in the center region of the channel for increasing $x$, thereby satisfying mass conservation. Hence, we consider the 3D data in the recovery region to be more physically realistic than the 2D data, and therefore the former are used in Figs. 14 and 21. 

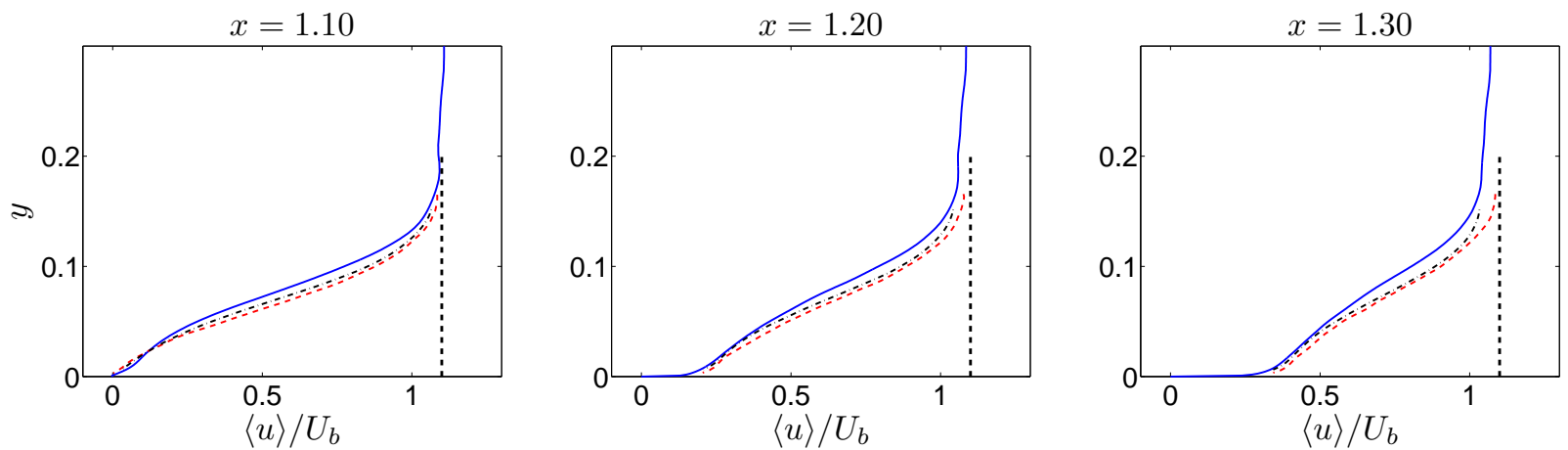

Figure 15. Hump flow: Mean velocity, $\langle\bar{u}\rangle$, downstream of reattachment. Vertical dashed thick lines are drawn at $\langle u\rangle / U_{b}=1.10$. _ : Prediction, baseline inlet fluctuations; - - - : 2D PIV experiments; - - : 3D PIV experiments.

Figure 16 presents the resolved and modelled Reynolds shear stresses. After the inflow section $(x=0.65)$, the resolved shear stress increases for increasing magnitude of inlet fluctuations, as expected. At $x=1.1$ and further downstream, the turbulent flow has nearly been re-established with little historical effect of inlet fluctuations, where different magnitudes of inflow turbulent fluctuations have produced similar levels of the resolved shear stresses. The modelled Reynolds stresses (which are shown with opposite sign to enhance readability) are negligible except at $x=0.65$ where in case of small inlet fluctuations they are comparable to the resolved stresses.
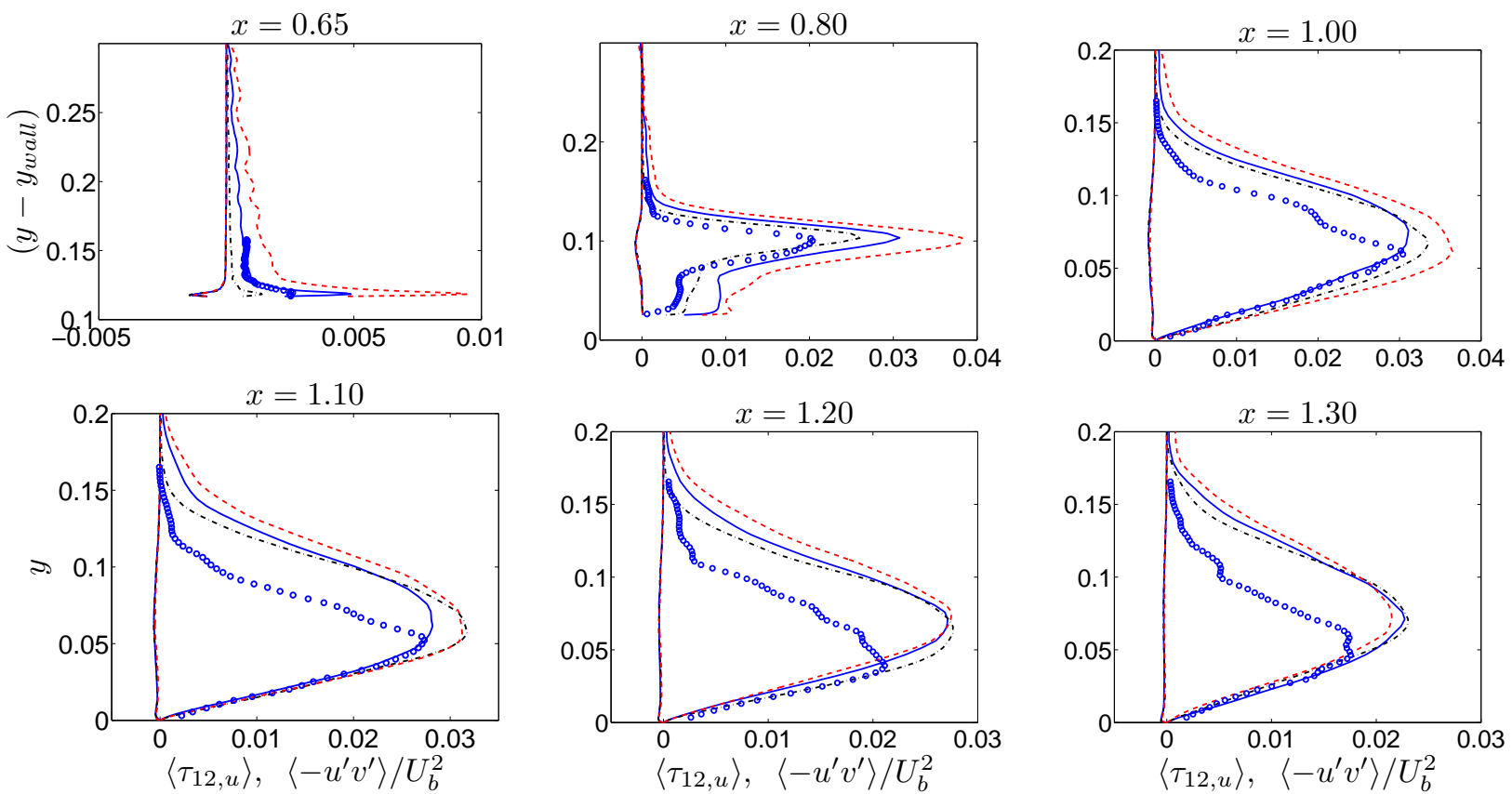

Figure 16. Hump flow: resolved and modelled turbulent shear stresses.

: baseline inlet fluctuations (see Fig. 4);

- - : $1.5 \times$ (baseline inlet fluctuations); - _ : $0.5 \times$ (baseline inlet fluctuations); $;$ : 2D PIV experiments. 
The turbulent viscosities depend only weakly on the magnitude of the inlet fluctuations, as shown in Fig. 17. Large inlet fluctuations do generate slightly large turbulent viscosities when the flow is re-adapting over a short distance after the inflow section. Although the ratio of the turbulent viscosities to the molecular viscosity is large (almost 70), Fig. 16 shows that the modelled shear stress is several orders of magnitude smaller than the resolved one.

It can be noted that in previous hump flow simulations by the present authors, ${ }^{29}$ the turbulent viscosities were up to $50 \%$ larger. Although a different discretization scheme was used in that work (pure central differencing), the main reason is that $\sigma_{k, u}=\sigma_{k}$ and $\sigma_{\varepsilon, u}=\sigma_{\varepsilon}$ were used (by mistake). This decreased the turbulent diffusion in the $k_{u}$ and $\varepsilon_{u}$ equations by a factor or $f_{k}^{-2}=6$ (since $f_{k}=0.4$ ) and consequently the peaks in $k_{k}$ and $\varepsilon_{u}$ were not smeared out by diffusion and the turbulent viscosity became large. This effect of setting $\sigma_{k, u}=\sigma_{k}$ and $\sigma_{\varepsilon, u}=\sigma_{\varepsilon}$ was discussed in Ref. 15 for fully developed channel flow and flow over periodic hills.
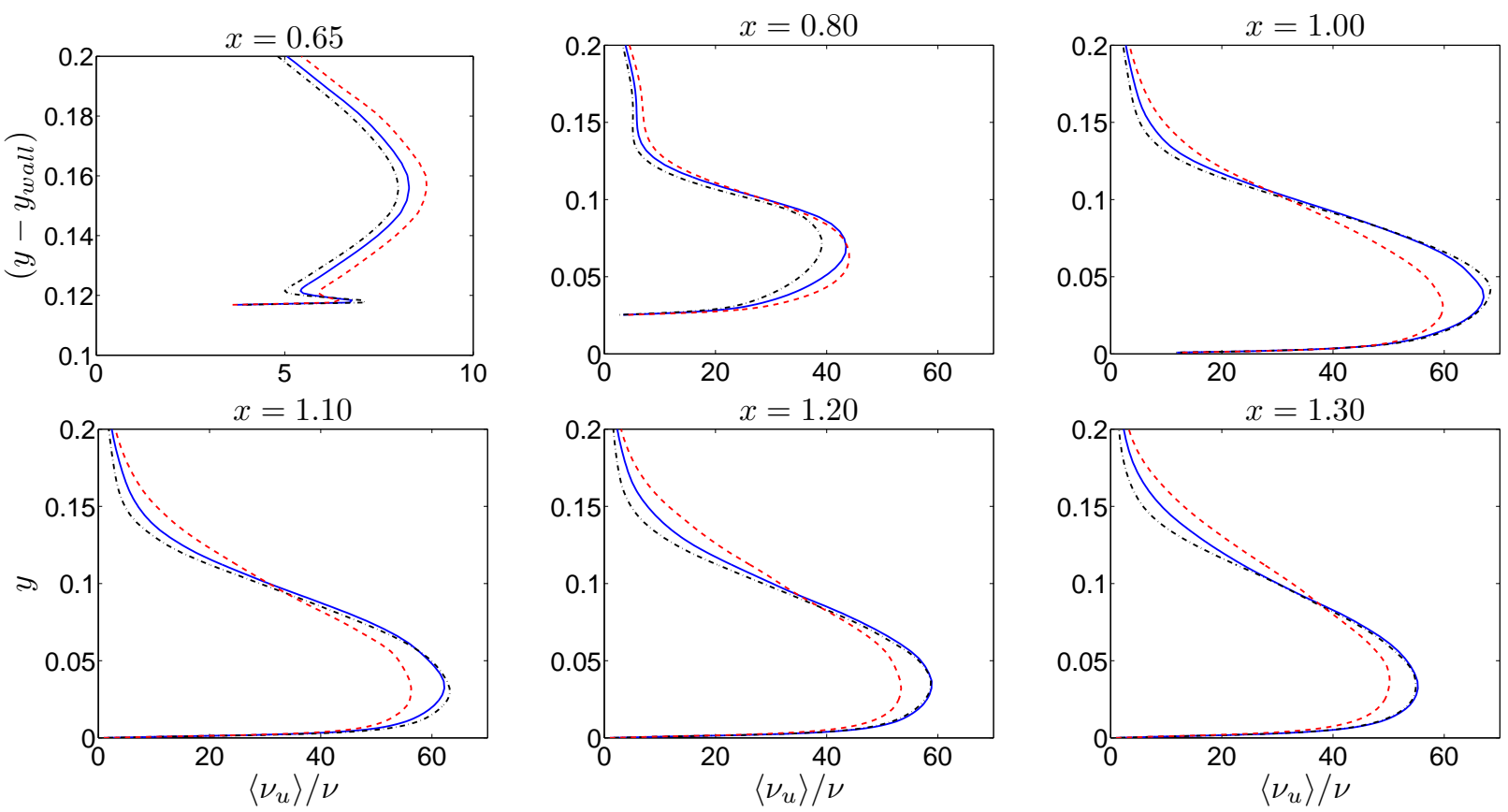

Figure 17. Hump flow: modelled turbulent eddy viscosity. : baseline inlet fluctuations (see Fig. 4); - - : $1.5 \times$ (baseline inlet fluctuations); - _ : $0.5 \times$ (baseline inlet fluctuations).

Figure 18 shows flow structures in the form of isosurface of $Q$. It can be seen that the turbulent scales are fairly large downstream of the recirculation region.

In Ref. 29 the influence of using different $f_{k}$ was investigated. Two additional simulations were carried 


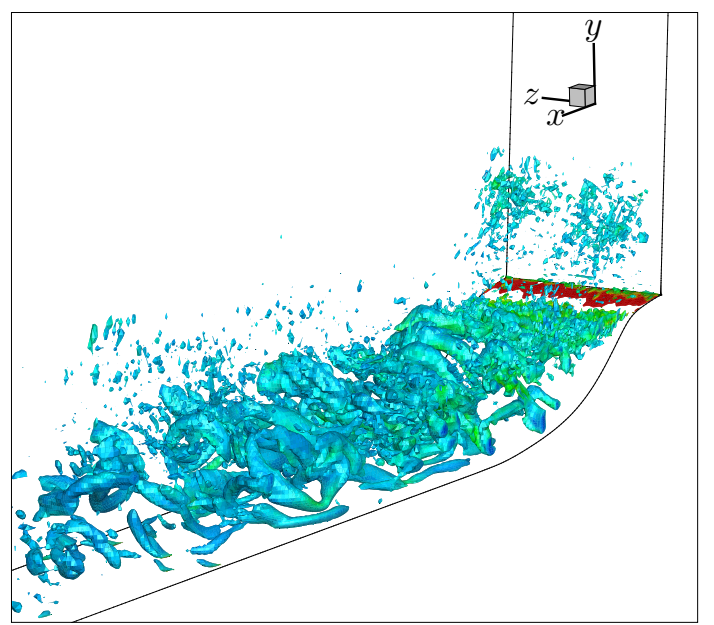

Figure 18. Hump flow: isosurface of $Q=-\frac{\partial \bar{u}_{i}}{\partial x_{j}} \frac{\partial \bar{u}_{j}}{\partial x_{i}}=500$. is shown and it is colored by vorticity magnitude. Flow from right to left.

out, one with $f_{k}=0.3$ and one with $f_{k}=0.5$. Due to space constraint, these results are not presented in this paper. The main conclusion was that the sensitivity was weak, but that an increase/decrease in $f_{k}$ gave - as expected - an increase/decrease in the turbulent viscosity.

The spanwise extent of the computational domain for all cases presented above was $Z_{\max }=0.2$. To investigate whether this is large enough, longitudinal spanwise two-point correlations are presented in Fig. 19. Two streamwise positions at which the two-point correlation was found to be the largest are shown, namely at $x=0.86$ and $x=2.56$. Three wall-normal locations are chosen. As can be seen, the two-point correlations do not always fall down to zero as they should. At $x=0.86$ and $y-y_{\text {wall }}=0.00085$, a negative correlation persists for a large separation distance, $\hat{z}$. It is slightly worse near the outlet at $x=2.56$. Both positive and negative correlations are found at a large separation distance of about $\hat{z}=0.1$. The integral length scale, $L_{\text {int }}$, was computed using the two-point correlation, and it was confirmed that the length scale is much larger far downstream in the flow than in the recirculation region. At $x=0.86$ and $x=2.56$, for example, $0.02<L_{\text {int }}<0.03$ and $0.04<L_{\text {int }}<0.06$, respectively. For comparison, the turbulent length scale, $L_{t}=k /\left(\omega c_{\mu}^{1 / 4}\right)$, from a 2D RANS using the $k-\omega$ model was computed and it was found to be much smaller, $L_{\text {int }} \simeq 0.01$ at both $x=0.86$ and $x=2.56$.

To further evaluate the possible effect of the spanwise extent, an additional simulation with $N_{k}=128$ is carried out by extending the spanwise size twice as large as the baseline case, i.e. $Z_{\max }=0.4$. The results are presented in Figs. 21 and 22. It can be seen that the results are very similar to the baseline simulations which 


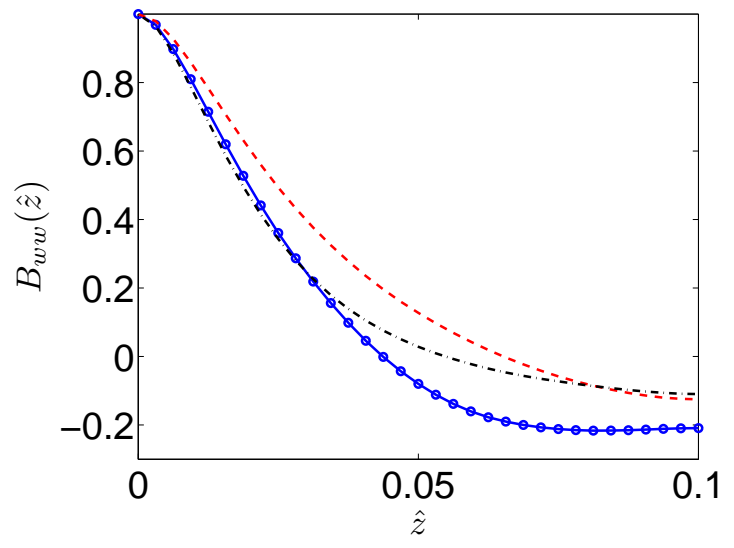

(a) $x=0.86$.

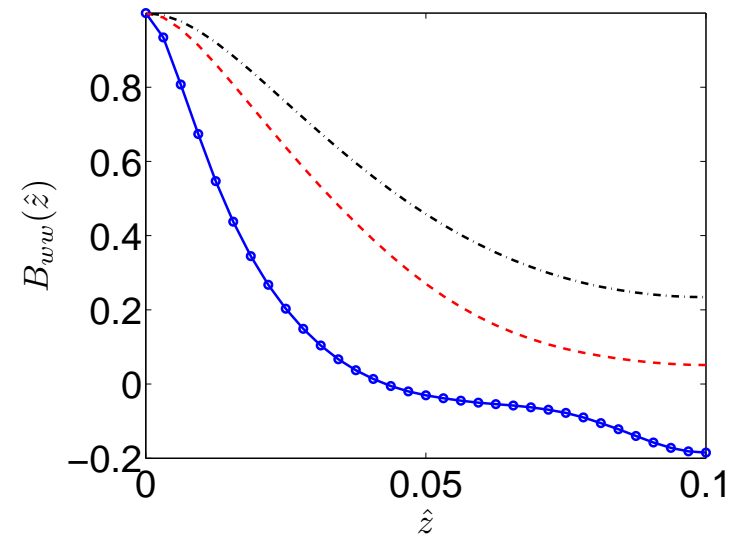

(b) $x=2.56$.

Figure 19. Hump flow. Two-point correlations. $: y-y_{\text {wall }}=0.00085 ;-\_-: y-y_{\text {wall }}=0.014 ; \ldots-\ldots: y-y_{\text {wall }}=0.10$. Markers indicate the grid spanwise resolution.

indicates that the baseline spanwise extent of $Z_{\max }=0.2$ is sufficient. Furthermore, in our previous study ${ }^{29}$ it was found that refining the mesh by a factor of two in the spanwise direction $\left(N_{k}=128, Z_{\max }=0.2\right)$ has no effect on the predicted results. This suggests further that the spanwise resolution in the baseline case is sufficiently fine.

Two additional sets of results are also included in these figures, one computed with the WALE model ${ }^{39}$ and one using pure central differencing for the momentum equations. Baseline inlet fluctuations are used for the two simulations using the PANS model, and better results were obtained with the WALE model by reducing the amplitude of inlet fluctuations by a factor of two.

Figure 20 presents the predicted pressure coefficient and the skin friction in comparison with the WALE model. As can be seen, the agreement with experiments is considerably worse than with the LRN PANS model. Nevertheless, the predicted velocity profiles are in fairly good agreement with experiments, but the profile at $x=1$ reveals the weak recirculation region which is also seen in the $C_{f}$ profile in Fig 20(b).

In the third simulation presented in Figs. 20-23 pure central differencing is used in the momentum equation. The wall pressure, velocity profiles and the turbulent viscosities are very similar to the those obtained with 5\% upwinding, see Figs. 13-17. The skin friction in Fig. 20(b) reveals a slightly stronger backflow with pure central differencing than with 5\% upwinding Fig. 13(b). However, the resolved shear stresses in Fig. 22 exhibit large unphysical resolved shear stress in the bulk flow region which are due to the 

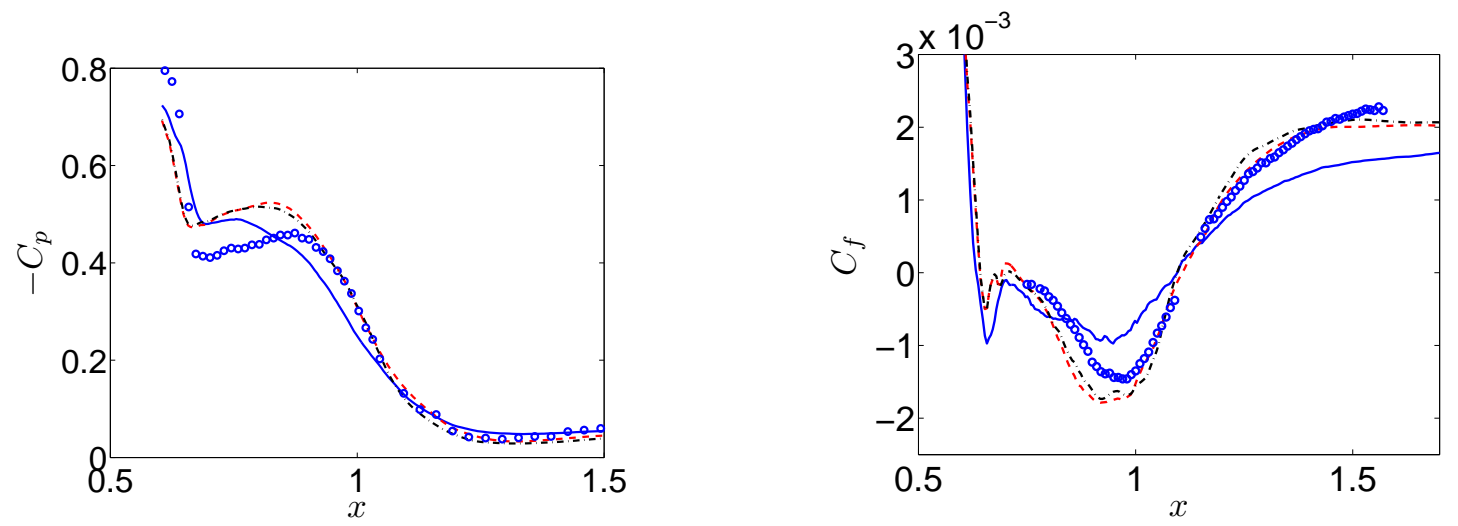

Figure 20. Hump flow. (a) Pressure coefficient; (b) Skin friction. _— : WALE model; --- : $N_{k}=128, Z_{m a x}=0.4$; -- - : Central differencing scheme; o: experiments.

use of the pure central differencing scheme. It was found in Ref. 29 that the unphysical fluctuations decrease with increasing amplitude of the synthetic inlet fluctuations. The reason is simply that central differencing works well in flow regions with resolved turbulence but not in regions with small (or no) resolved turbulence. However, in the outer region $(y>0.3)$ there are no unphysical oscillations. This is probably due to the fact that the mean velocity gradients are negligible in this region and hence no oscillations are triggered. It can be seen that, already at $x=0.8$, the unphysical fluctuations at $y<0.3$ have disappeared.

It should be mentioned that a similar finding was made in Ref. 40 when using LES for flow around an airfoil. When pure central differencing was used, large numerical, unphysical fluctuations were present in the inviscid region. In the regions where the large-scale turbulence was resolved by LES, however, no numerical oscillations were present.

One simulation was carried out using $20 \%$ upwinding. The predicted velocity profile showed somewhat worse agreement with experiments (not shown) than when using $5 \%$ upwinding. The magnitude of the resolved shear stresses was slightly smaller in the recirculation zone (the peak at $x=0.8$, for example, was approximately $5 \%$ smaller than the baselline case in Fig. 16). The largest difference was seen in the skin friction where, for example, the peak in the recirculation reached a value of -0.0020 compared to -0.0016 for the baseline case (see Fig. 13(b)). 

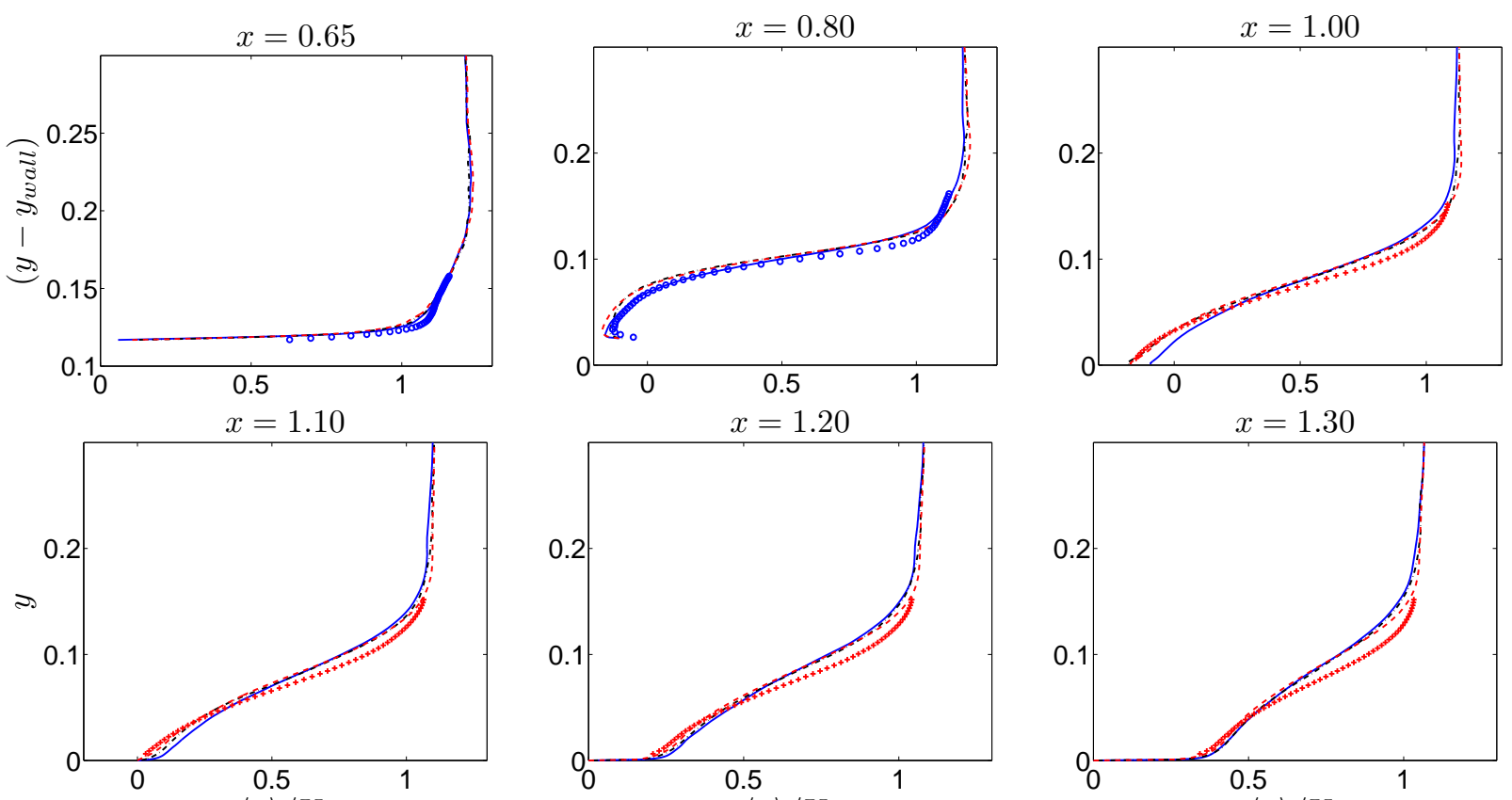

$\langle u\rangle / U_{b}$

$\langle u\rangle / U_{b}$

$\langle u\rangle / U_{b}$

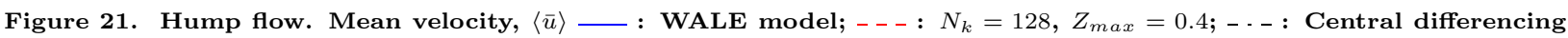
scheme; ; ०: 2D PIV experiments; +: 3D PIV experiments.
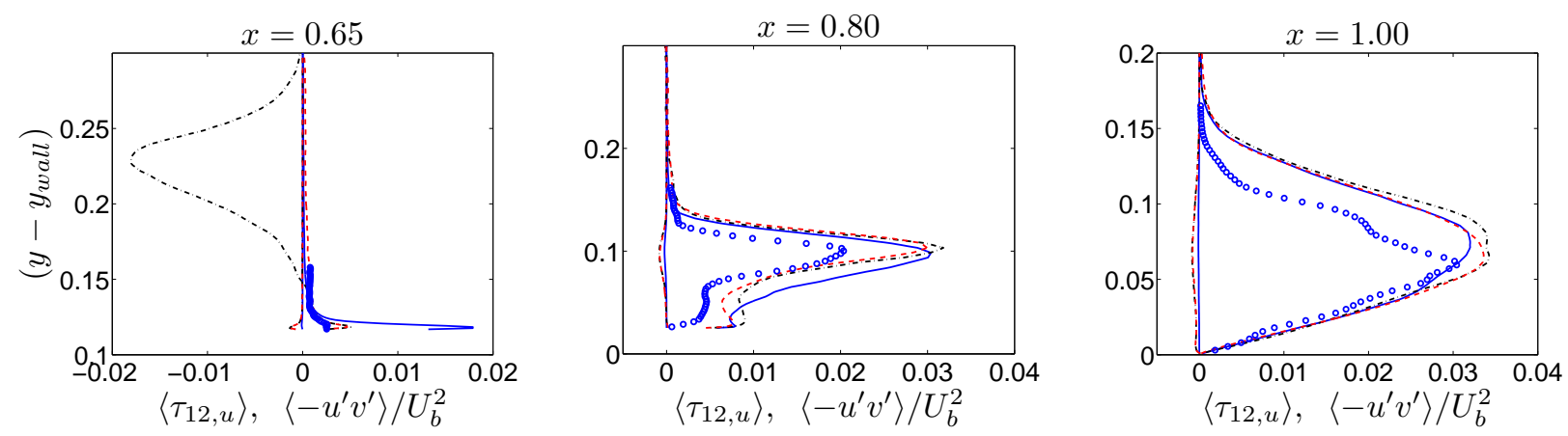

Figure 22. Hump flow. Resolved and modelled shear stresses. — : WALE model; - - - : $N_{k}=128, Z_{\max }=0.4$; - . - : Central differencing scheme; ०: 2D PIV experiments.

\section{Summary and Concluding Remarks}

By adapting the model coefficient (typically, $f_{k}$ ) and the grid resolution, the PANS approach may function as a RANS model or as an LES model. Using this inherent modelling mechanism, a PANS-based embedded LES method is presented. By setting $f_{k}^{R A N S}=1$ in the RANS region, the PANS formulation returns to its RANS base model, and in the LES region a smaller value of $f_{k}$ is used (baseline value $f_{k}^{L E S}=0.4$ ). Along with the presentation of the modelling method and its verification, an emphasis in the present work has been placed on the effect of synthetic anisotropic fluctuations imposed at the RANS-LES interface. The method has been verified in computations of turbulent channel flow and hump flow. 

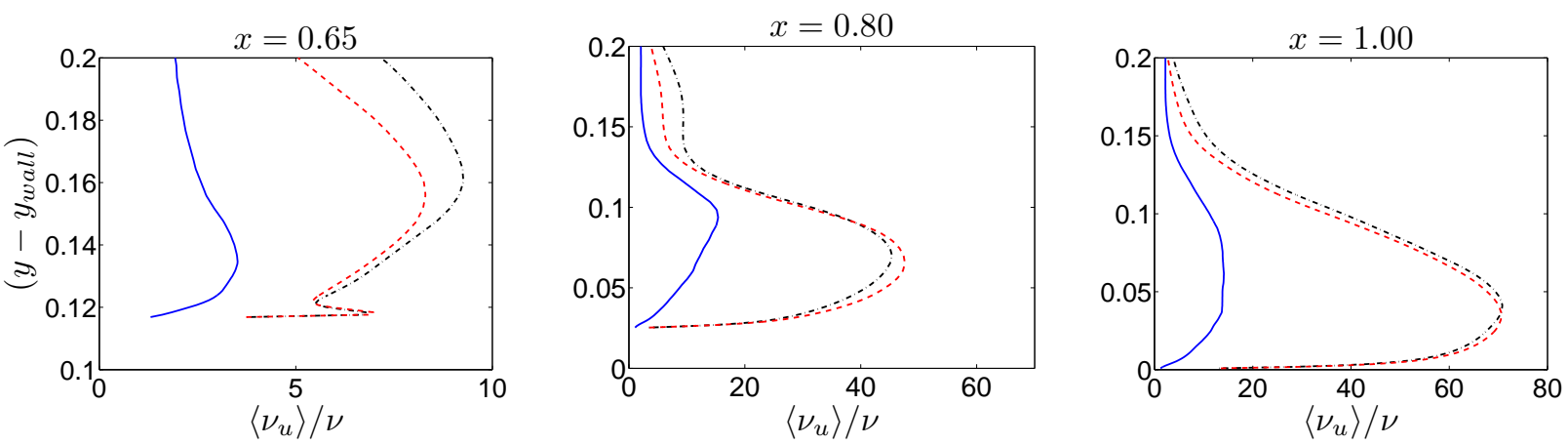

Figure 23. Hump flow. Turbulent viscosity. __ : WALE model; - - - : $N_{k}=128, Z_{\max }=0.4$; - - - : Central differencing scheme.

Investigation on the effect of the domain extent in the spanwise direction was conducted by doubling the spanwise extent of the computations domain. It is confirmed that the domain size is adequate in the baseline configuration and in a previous study ${ }^{29}$ it has been concluded that also the spanwise grid resolution is sufficient.

For the channel flow, it was found that the addition of anisotropic fluctuations at the RANS-LES interface is very effective to force an efficient re-establishment towards fully developed resolved turbulence and, consequently, enabling reasonably resolved turbulent fluctuations in the downstream LES region. Already at two half-channel widths downstream of the interface, the resolved turbulence agrees rather well with DNS data, and the wall friction velocity has reached $99 \%$ of its fully developed value. The treatment of the modelled $k_{u}$ and $\varepsilon_{u}$ across the interface is important. New "inlet" (to the LES region) values of $k_{u}$ and $\varepsilon_{u}$ were prescribed at the interface by setting the usual convection and diffusion at the interface to zero and introducing sources that correspond to convection and diffusion of interface values, $k_{\text {inter }}$ and $\varepsilon_{\text {inter }}$, into the LES region. The former was set to $f_{k}^{L E S} k_{R A N S}$ and the latter to $C_{\mu}^{3 / 4} k_{\text {inter }}^{3 / 2} / \ell_{s g s}$ where $\ell_{s g s}=C_{S} \Delta$ was taken from the Smagorinsky model and a baseline value of $C_{S}=0.07$ is specified. Different values of $C_{S}$ were evaluated and were found to have noticeable effects on the predicted results. Finally, different values of $f_{k}^{L E S}$ were tested. It was found that for $0.2 \leq f_{k}^{L E S} \leq 0.5$ the impact of $f_{k}^{L E S}$ was insignificant.

The RANS region and the LES region were computed concurrently in the channel flow simulations. In the hump flow simulations, however, the entire flow was first simulated with 2D RANS. The 2D RANS results at $x=0.6$ (60\% of the hump length) were then used to prescribe the time-averaged (mean) inflow conditions 
for the LES simulation. Anisotropic synthetic fluctuations were added at the LES inlet, and the $k_{u}$ and $\varepsilon_{u}$ were prescribed in the same way as in the channel flow simulations. The embedded LES method was found to give good results for this flow as well. The effect of increased and decreased magnitude of the anisotropic synthetic inlet fluctuations was investigated. It was found that the prediction of the separation bubble on the lee side of the hump is somewhat affected by the magnitude of inflow fluctuations imposed, in relation to the resolved turbulent diffusion in the free shear layer above the separation bubble. With too small, or with no, inflow fluctuations, the predicted reattachment after the recirculation bubble becomes delayed because the resolved turbulent diffusion is too small and the free shear layer above the recirculation region becomes less diffusive.

Simulations using the WALE model were also carried out of the hump flow to compare this model with the LRN PANS model in the embedded LES region. It was found that the WALE model gave degraded agreement with the experiment, as compared with the LRN PANS model. The turbulent viscosities obtained with the WALE model were much smaller than those obtained with the LRN PANS model. This suggests that LRN PANS model, when used in its turbulence-resolving mode, is able to give better results on coarser meshes than conventional LES using classical SGS models.

\section{Acknowledgments}

The financial support of SNIC (the Swedish National Infrastructure for Computing) for computer time at C3SE (Chalmers Center for Computational Science and Engineering) is gratefully acknowledged. This project was financed by the EU project ATAAC (Advanced Turbulence Simulation for Aerodynamic Application Challenges), Grant Agreement No. 233710, and the Chalmers Sustainable Transport Initiative. The ATAAC project is described at http://cfd.mace.manchester.ac.uk/ATAAC/WebHome

\section{References}

\footnotetext{
${ }^{1}$ Quéméré, P. and Sagaut, P., "Zonal multi-domain RANS-LES simulations of turbulent flows," International Journal for Numerical Methods in Fluids, Vol. 40, No. 7, 2002, pp. 903-925.

${ }^{2}$ Batten, P., Goldberg, U., and Chakravarthy, S., "Interfacing Statistical Turbulence Closures with Large-Eddy Simulation," AIAA Journal, Vol. 42, No. 3, 2004, pp. 485-492.

${ }^{3}$ Terracol, M., "A Zonal RANS/LES Approach for Noise Sources Prediction," Flow, Turbulence and Combustion, Vol. 77,
} 
No. 1-4, 2006, pp. 161-184.

${ }^{4}$ Mathey, F. and Cokljat, D., "Zonal Multi-domain RANS/LES Simulation of Air Flow over the Ahmed Body," Engineering Turbulence Modelling and Measurements 6, edited by W. Rodi and M. Mulas, Elsevier, 2005, pp. 647-656.

${ }^{5}$ Jarrin, N., Prosser, R., Uribe, J.-C., , Benhamadouche, S., and Laurence, D., "Reconstruction of turbulent fluctuations for hybrid RANS/LES simulations using a Synthetic-Eddy Method," International Journal of Heat and Fluid Flow, Vol. 30, 2009, pp. 435-442.

${ }^{6}$ Mary, I., "Non-DES Type Hybrid RANS-LES Modelling Approaches," Notes on Numerical Fluid Mechanics and Multidisciplinary Design, Vol. 111, 2010, pp. 205-212.

${ }^{7}$ Zhang, Q., Schröder, W., and Meinke, M., "A zonal RANS-LES method to determine the flow over a high-lift configuration," Computers \& Fluids, Vol. 39, No. 7, 2010, pp. 1241-1253.

${ }^{8}$ Ma, J., Davidson, L., Peng, S.-H., and Wang, F., "Partially Averaged Navier-Stokes Model Modelling of Turbulent Channel Flow with and without Forcing," 6th International Symposium on Turbulence, Heat and Mass Transfer, edited by K. Hanjalic, Y. Nagano, and S. Jakirlic, 2009.

${ }^{9}$ Davidson, L., "Hybrid LES-RANS: back scatter from a scale-similarity model used as forcing," Phil. Trans. of the Royal Society A, Vol. 367, No. 1899, 2009, pp. 2905-2915.

${ }^{10}$ Shur, M. L., Spalart, P., Strelets, M. K., and Travin, A., "A Rapid and Accurate Switch from RANS to LES in Boundary Layers Using an Overlap Region," Flow, Turbulence and Combustion, Vol. 86, 2011, pp. 179-206.

${ }^{11}$ Girimaji, S., "Partially-Averaged Navier-Stokes Model for Turbulence: Implementation and Validation," 43rd AIAA Aerospace Sciences Meeting and Exhibt, 10-13 January, Reno, Nevada, 2005.

${ }^{12}$ Girimaji, S., "Partially-Averaged Navier-Stokes Model for Turbulence: A Reynolds-Averaged Navier-Stokes to Direct Numerical Simulation Bridging Method," ASME Journal of Applied Mechanics, Vol. 73, No. 2, 2006 , pp. 413-421.

${ }^{13}$ Girimaji, S., Jeong, E., and Srinivasan, R., "Partially-Averaged Navier-Stokes Method for Turbulence: Fixed Point Analysis and Comparison With Unsteady Partially Averaged Navier-Stokes," ASME Journal of Applied Mechanics, Vol. 73, No. 2, 2006, pp. 422-429.

${ }^{14}$ Basara, B., Krajnović, S., Girimaji, S., and Pavlović, Z., "Near-Wall Formulation of the Partially Averaged Navier Stokes Turbulence Model," AIAA Journal, Vol. 49, No. 12, 2011, pp. 2627-2636.

${ }^{15}$ Ma, J., Peng, S.-H., Davidson, L., and Wang, F., "A Low Reynolds Number Variant of Partially-Averaged Navier-Stokes Model for Turbulence," International Journal of Heat and Fluid Flow, Vol. 32, 2011, pp. 652-669.

${ }^{16}$ Abe, K., Kondoh, T., and Nagano, Y., "A New Turbulence Model for Predicting Fluid Flow and Heat Transfer in Separating and Reattaching Flows - 1. Flow Field Calculations," Int. J. Heat Mass Transfer, Vol. 37, 1994, pp. 139-151.

${ }^{17}$ Schiestel, R. and Dejoan, A., "Towards a new partially integrated transport model for coarse grid and unsteady turbulent flow simulations," Theoretical and Computational Fluid Dynamics, Vol. 18, 2005, pp. 443-468.

${ }^{18}$ Davidson, L., "A New Approach of Zonal Hybrid RANS-LES Based on a Two-equation $k-\varepsilon$ Model," ETMM9: International ERCOFTAC Symposium on Turbulence Modelling and Measurements, Thessaloniki, Greece, 2012. 
${ }^{19}$ Davidson, L., "Large Eddy Simulation of Heat Transfer in Boundary layer and Backstep Flow Using PANS (to be presented)," Turbulence, Heat and Mass Transfer, THMT-12, Palermo, Sicily/Italy, 2012.

${ }^{20}$ Billson, M., Eriksson, L.-E., and Davidson, L., "Modeling of Synthetic Anisotropic Turbulence and its Sound Emission," The 10th AIAA/CEAS Aeroacoustics Conference, AIAA 2004-2857, Manchester, United Kindom, 2004.

${ }^{21}$ Billson, M., Computational Techniques for Turbulence Generated Noise, Ph.D. thesis, Dept. of Thermo and Fluid Dynamics, Chalmers University of Technology, Göteborg, Sweden, 2004.

${ }^{22}$ Davidson, L. and Billson, M., "Hybrid LES/RANS Using Synthesized Turbulence for Forcing at the Interface," International Journal of Heat and Fluid Flow, Vol. 27, No. 6, 2006, pp. 1028-1042.

${ }^{23}$ Moser, R., Kim, J., and Mansour, N., "Direct Numerical Simulation of Turbulent Channel Flow up to Re $\tau=590$," Physics of Fluids, Vol. 11, 1999, pp. 943-945.

${ }^{24}$ http://www.tfd.chalmers.se/ lada/projects/inlet-boundary-conditions/proright.html.

${ }^{25}$ Davidson, L., "HYBRID LES-RANS: Inlet Boundary Conditions for Flows With Recirculation," Advances in Hybrid RANS-LES Modelling, Vol. 97 of Notes on Numerical Fluid Mechanics and Multidisciplinary Design, Springer Verlag, 2008, pp. $55-66$.

${ }^{26}$ Davidson, L. and Peng, S.-H., "Hybrid LES-RANS: A one-equation SGS Model combined with a $k-\omega$ model for predicting recirculating flows," International Journal for Numerical Methods in Fluids, Vol. 43, 2003, pp. 1003-1018.

${ }^{27}$ Emvin, P., The Full Multigrid Method Applied to Turbulent Flow in Ventilated Enclosures Using Structured and Unstructured Grids, Ph.D. thesis, Dept. of Thermo and Fluid Dynamics, Chalmers University of Technology, Göteborg, 1997.

${ }^{28}$ van Leer, B., "Towards the Ultimate Conservative Difference Scheme. Monotonicity and Conservation Combined in a Second Order Scheme," Journal of Computational Physics, Vol. 14, 1974, pp. 361-370.

${ }^{29}$ Davidson, L. and Peng, S.-H., "Emdedded LES with PANS," 6th AIAA Theoretical Fluid Mechanics Conference, AIAA paper 2011-3108, 27-30 June, Honolulu, Hawaii, 2011.

${ }^{30}$ Patankar, S., Numerical Heat Transfer and Fluid Flow, McGraw-Hill, New York, 1980.

${ }^{31}$ Welty, J., C.E.Wicks, and Wilson, R., Fundamentals of Momentum, Heat, and Mass Transfer, John Wiley \& Sons, New York, 3rd ed., 1984.

${ }^{32}$ Hoyas, S. and Jimenez, J., "Reynolds number effects on the Reynolds-stress budgets in turbulent channels," Physics of Fluids A, Vol. 20, No. 101511, 2008.

${ }^{33}$ Keating, A., Piomelli, U., Balaras, E., and Kaltenbach, H.-J., "A priori and a posteriori tests of inflow conditions for large-eddy simulation," Physics of Fluids A, Vol. 16, No. 12, 2004, pp. 4696-4712.

${ }^{34}$ Adamian, D. Y. and Travin, A., "Improved Version of the Synthetic Eddy Method for Setting Nonstationary Inflow Boundary Conditions in Calculating Turbulent Flows," High Temperature, Vol. 49, No. 5, 2011, pp. 704-711.

${ }^{35}$ Avdis, A., Lardeau, S., and Leschziner, M., "Large Eddy Simulation of Separated Flow over a Two-dimensional Hump with and without Control by Means of a Synthetic Slot-jet," Flow, Turbulence and Combustion, Vol. 83, No. 3, 2009, pp. 343370 . 
${ }^{36}$ Saric, S., Jakirlic, S., Djugum, A., and Tropea, C., "Computational analysis of locally forced flow over a wall-mounted hump at high-Re number," International Journal of Heat and Fluid Flow, Vol. 27, 2006, pp. 707-720.

${ }^{37}$ Greenblatt, D., Paschal, K., Yao, C.-S., Harris, J., Schaeffler, N. W., and Washburn, A., "A Separation Control CFD Validation Test Case. Part 1: Baseline \& Steady Suction," AIAA-2004-2220, 2004.

${ }^{38}$ Greenblatt, D., Paschal, K., Yao, C.-S., and Harris, J., "A Separation Control CFD Validation Test Case Part 1: Zero Efflux Oscillatory Blowing," AIAA-2005-0485, 2005.

${ }^{39}$ Nicoud, F. and Ducros, F., "Subgrid-scale stress modelling based on the square of the velocity gradient tensor," Flow, Turbulence and Combustion, Vol. 62, 1999, pp. 183-200.

${ }^{40}$ Dahlström, S. and Davidson, L., "Large Eddy Simulation of the Flow Around an Aerospatiale A-aerofoil," ECCOMAS 2000, European Congress on Computational Methods in Applied Sciences and Engineering, 11-14 September, Barcelona, Spain, 2000 . 